\title{
Itaconate and fumarate derivatives exert a dual inhibitory effect on canonical NLRP3 activation in macrophages and microglia
}

1 Christopher Hoyle*, Jack P Green, Stuart M Allan, David Brough*, Eloise Lemarchand

2 Geoffrey Jefferson Brain Research Centre, The Manchester Academic Health Science Centre,

3 Northern Care Alliance NHS Group, University of Manchester

4 Division of Neuroscience and Experimental Psychology, School of Biological Sciences, Faculty of

5 Biology, Medicine and Health, University of Manchester, AV Hill Building, Manchester, UK

6 The Lydia Becker Institute of Immunology and Inflammation, University of Manchester, AV Hill

7 Building, Manchester, UK

8 * Corresponding authorship:

9 Christopher Hoyle

10 christopher.hoyle@manchester.ac.uk

11 ORCID iD: https://orcid.org/0000-0002-2075-303X

12 David Brough

13 david.brough@manchester.ac.uk

14 ORCID iD: https://orcid.org/ 0000-0002-2250-2381

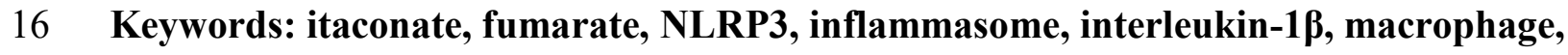

17 microglia, organotypic hippocampal slice culture 
bioRxiv preprint doi: https://doi.org/10.1101/2021.02.01.429180; this version posted February 2, 2021. The copyright holder for this preprint (which was not certified by peer review) is the author/funder, who has granted bioRxiv a license to display the preprint in perpetuity. It is made available under aCC-BY 4.0 International license.

\section{Summary statement}

19 We show that itaconate and fumarate derivatives inhibit both the priming and activation steps of

20 NLRP3 inflammasome responses in macrophages and microglia, revealing the importance of 21 immunometabolic NLRP3 regulation. 


\section{Abstract}

23 The NLRP3 inflammasome is a multi-protein complex that regulates the protease caspase-1 and 24 subsequent interleukin (IL)-1 $\beta$ release from cells of the innate immune system, or microglia in the 25 brain, in response to infection or injury. Derivatives of the metabolites itaconate and fumarate, dimethyl 26 itaconate (DMI), 4-octyl itaconate (4OI) and dimethyl fumarate (DMF), limit both expression of IL-1 $\beta$, and IL-1 $\beta$ release following NLRP3 inflammasome activation. However, the direct effects of these metabolite derivatives on NLRP3 inflammasome responses in macrophages and microglia require further investigation. Using murine bone marrow-derived macrophages, mixed glia and organotypic

30 hippocampal slice cultures (OHSCs), we demonstrate that DMI and 4OI pre-treatment limited IL-1 $\beta$,

31 IL-6 and tumor necrosis factor production in response to lipopolysaccharide (LPS) priming, as well as

32 inhibiting subsequent NLRP3 inflammasome activation. DMI, 4OI, DMF and monomethyl fumarate 33 (MMF), another fumarate derivative, also directly inhibited biochemical markers of NLRP3 activation 34 in LPS-primed macrophages, mixed glia and OHSCs, including ASC speck formation, caspase-1 35 activation, gasdermin D cleavage and IL-1 $\beta$ release. Finally, DMF, an approved treatment for multiple 36 sclerosis, as well as DMI, 4OI and MMF, inhibited NLRP3 activation in macrophages in response to 37 the phospholipid lysophosphatidylcholine, which is used to induce demyelination, suggesting a 38 possible mechanism of action for DMF in multiple sclerosis through NLRP3 inhibition. Together, these 39 findings reveal the importance of immunometabolic regulation for both the priming and activation 40 steps of NLRP3 activation in macrophages and microglia. Furthermore, we highlight itaconate and 41 fumarate derivatives as a potential therapeutic option in NLRP3-driven diseases, including in the brain. 


\section{Introduction}

43 Macrophages are innate immune effector cells that regulate inflammatory responses upon infection or tissue injury to restore tissue homeostasis by promoting pathogen death or tissue and wound repair. In the brain, macrophage-like cells called microglia are important effectors of this inflammatory response. Inflammasomes are cytosolic complexes that regulate the inflammatory response in immune cells and microglia. In particular, the nucleotide-binding oligomerisation domain-, leucine-rich repeat- and pyrin domain-containing protein 3 (NLRP3) inflammasome has been implicated in a range of noncommunicable diseases that are characterised by an inflammatory response (Chen and Nuñez, 2010; Rock et al., 2010). Although several pathways of NLRP3 activation have been described (Gaidt et al., 2016; Kayagaki et al., 2011), canonical NLRP3 activation is the most studied. The canonical pathway consists of an initial priming step, typically mediated through Toll-like receptor signaling, that upregulates NLRP3 and IL-1 $\beta$ expression, followed by a subsequent NLRP3 activating stimulus. A broad range of pathogen- or damage-associated molecular patterns are known to act as this activating stimulus, including the potassium ionophore nigericin, extracellular ATP (Perregaux and Gabel, 1994), amyloid- $\beta$ aggregates (Halle et al., 2008) or silica crystals (Dostert et al., 2008; Hornung et al., 2008).

The precise mechanism by which these stimuli induce NLRP3 activation is still unclear, with potassium efflux-dependent (Muñoz-Planillo et al., 2013) and -independent (Groß et al., 2016) mechanisms suggested to elicit dispersal of the trans-Golgi network, leading to inflammasome formation (Chen and Chen, 2018). We recently proposed organelle dysfunction to be a crucial cellular event that leads to NLRP3 activation (Seoane et al., 2020). Once activated, NLRP3 interacts with the adaptor protein ASC (apoptosis-associated speck-like protein containing a caspase recruitment domain), causing the formation of an ASC speck that drives activation of the inflammasome effector protein caspase-1 (Boucher et al., 2018; Schroder and Tschopp, 2010). Active caspase-1 then cleaves gasdermin D and pro-interleukin (IL)-1 $\beta$, with gasdermin D pores potentially forming the conduit for mature IL-1 $\beta$ release (He et al., 2015).

Immunometabolism has emerged as a regulator of macrophage inflammasome responses (O’Neill and Artyomov, 2019). Lipopolysaccharide (LPS) treatment of macrophages causes a metabolic shift from oxidative phosphorylation to glycolysis that is necessary for IL-1 $\beta$ production (Tannahill et al., 2013). Certain metabolites such as itaconate (O’Neill and Artyomov, 2019), succinate (Mills and O’Neill, 2014) and fumarate (Humphries et al., 2020) have immunoregulatory functions. For example, itaconate 
73 fumarate (DMF), are able to activate nuclear factor erythroid 2-related factor 2 (NRF2) signalling by

74 alkylating and subsequently inducing the degradation of the cytoplasmic NRF2 inhibitor kelch-like 75 ECH-associated protein 1 (KEAP1) (Mills et al., 2018). NRF2 is then able to translocate to the nucleus, where it not only upregulates the transcription of its target genes, but also prevents the recruitment of RNA polymerase II to NF- $\kappa$ B secondary response genes such as IL-6 and IL-1 $\beta$ (Kobayashi et al., 2016). DMI and 4OI also induce electrophilic stress and glutathione depletion in macrophages, which inhibits the LPS-induced translation of I $\mathrm{B} \zeta$ independently of NRF2, and this subsequently limits the expression of $\mathrm{I} \kappa \mathrm{B} \zeta$-dependent NF- $\kappa \mathrm{B}$ secondary response genes (Bambouskova et al., 2018; Swain et al., 2020). It must be acknowledged that the properties of these itaconate derivatives may not fully reflect the properties of endogenous itaconate (Swain et al., 2020). In vivo evidence also indicates the importance of itaconate responses, as mice deficient in $\operatorname{Irg} 1$, which therefore cannot produce itaconate, rapidly succumb to Mycobacterium tuberculosis infection, whereas there was no mortality in wild-type control mice (Nair et al., 2018). Interestingly, 4OI and DMF exhibit anti-viral and anti-inflammatory effects through NRF2 signalling in response to Severe Acute Respiratory Syndrome Coronavirus 2 (SARS-CoV2) infection (Olagnier et al., 2020).

Although itaconate derivatives are known to limit IL-1 $\beta$ expression, the direct effects of itaconaterelated compounds on NLRP3 inflammasome activation are less characterized. Previous studies suggest that DMF, an approved treatment for relapsing-remitting multiple sclerosis, and its metabolite monomethyl fumarate (MMF), limit NLRP3 inflammasome activation, with DMF exhibiting greater potency (Liu et al., 2016; Miglio et al., 2015). DMF has also been shown to directly succinate a cysteine residue on gasdermin D to limit pyroptotic cell death in response to NLRP3 activation in vitro and in vivo, but without inhibiting NLRP3 activation itself (Humphries et al., 2020). Itaconate and its derivative 4OI can inhibit NLRP3 activation in an NRF2-independent manner, through the modification of specific cysteine residues on NLRP3, which may prevent NLRP3's interaction with NEK7 and subsequent activation (Hooftman et al., 2020; Swain et al., 2020). IRG1-deficient macrophages, which cannot synthesize endogenous itaconate, exhibit enhanced IL-1 $\beta$ release in response to NLRP3 inflammasome activation. Finally, 4OI is effective at inhibiting NLRP3 activation in vivo (Hooftman et al., 2020).

101 Despite recent advances, further characterisation of the effects of itaconate- and fumarate-related 102 compounds on NLRP3 inflammasome activation is required in order to evaluate their therapeutic 103 potential. The relevance of immunometabolic regulation of microglial inflammasome responses in the 
104 brain is also unclear. Here, we demonstrate that itaconate derivative pre-treatment not only prevented

105 expression of IL-1 $\beta$, but also inhibited canonical NLRP3 inflammasome activation. We identified that

106 itaconate and fumarate derivatives were able to directly inhibit canonical NLRP3 inflammasome

107 activation, independent of their inhibitory effect on priming. These effects were consistent in mixed

108 glia and organotypic hippocampal slice cultures (OHSCs), two brain-relevant NLRP3 inflammasome

109 models (Hoyle et al., 2020). Finally, itaconate and fumarate derivatives inhibited NLRP3 activation

110 induced by lysophosphatidylcholine (LPC), a lipid molecule used to induce demyelination in models

111 of multiple sclerosis, further highlighting a potential mechanism of DMF action in multiple sclerosis

112 treatment. These findings reveal a dual anti-inflammatory effect of itaconate and fumarate derivatives

113 in the innate immune system and brain, through regulation of both the priming and activation steps of

114 canonical NLRP3 inflammasome responses. 


\section{Results}

117 Pre-treatment with itaconate and fumarate derivatives reduces NLRP3 priming and canonical

118 inflammasome activation

119 BMDMs were pre-treated with two cell-permeable derivatives of itaconate, DMI and 4OI, as well as

120 the fumarate derivative DMF, and the effects on NLRP3 priming and activation were assessed. DMI

121 and 4OI treatment alone did not induce NRF2 accumulation in murine WT BMDMs, but both enhanced

122 LPS-induced NRF2 accumulation (Figure 1A). DMF treatment was toxic to the cells at this treatment

123 duration and concentration, explaining the lack of NRF2 accumulation (Figure 1A, Supplementary

124 Figure 1). I $\kappa$ B $\zeta$ protein levels were not measured. DMI and 4OI pre-treatment inhibited the production

125 of pro-IL-1 $\beta$ in response to LPS priming, and whilst DMI reduced NLRP3 expression to a lesser extent,

126 4OI had no effect on NLRP3 levels (Figure 1A). No pro-IL-1 $\beta$ or NLRP3 protein was observed

127 following DMF pre-treatment, although this was probably due to toxicity prior to LPS priming (Figure

128 1A, Supplementary Figure 1). DMI and 4OI also strongly reduced LPS-induced IL-6 release, with a

129 smaller reduction in TNF release (Figure 1Bi, Bii). Consistent with inhibition of pro-IL-1 $\beta$ expression,

130 DMI and 4OI pre-treatment blocked IL-1 $\beta$ release in response to subsequent stimulation with LPS and

131 nigericin (Figure 1Biii). These data confirmed previous findings that itaconate derivative treatment

132 prior to LPS stimulation could limit inflammatory priming via NRF2 activation (Mills et al., 2018).

133 We next investigated whether itaconate derivative pre-treatment could reduce NLRP3 inflammasome

134 activation, as has been recently suggested (Swain et al., 2020). Treatment of murine BMDMs from

135 ASC-citrine reporter mice (Tzeng et al., 2016) with DMI, 4OI and DMF prior to LPS priming inhibited

136 the formation of ASC specks upon subsequent nigericin treatment (Figure 1Ci, Cii), with representative

137 images from this experiment after 90 minutes of nigericin treatment shown (Figure 1Ciii).

138 Morphological changes due to nigericin treatment are also shown using bright-field microscopy

139 (Supplementary Figure 1). DMF pre-treatment induced a high level of cell toxicity, perhaps explaining

140 its strong inhibitory effect on NLRP3 inflammasome formation and IL-1 $\beta$ release. These data

141 suggested that itaconate derivative pre-treatment may additionally inhibit the NLRP3 inflammasome

142 activation step, as well as inhibiting the priming stage. 


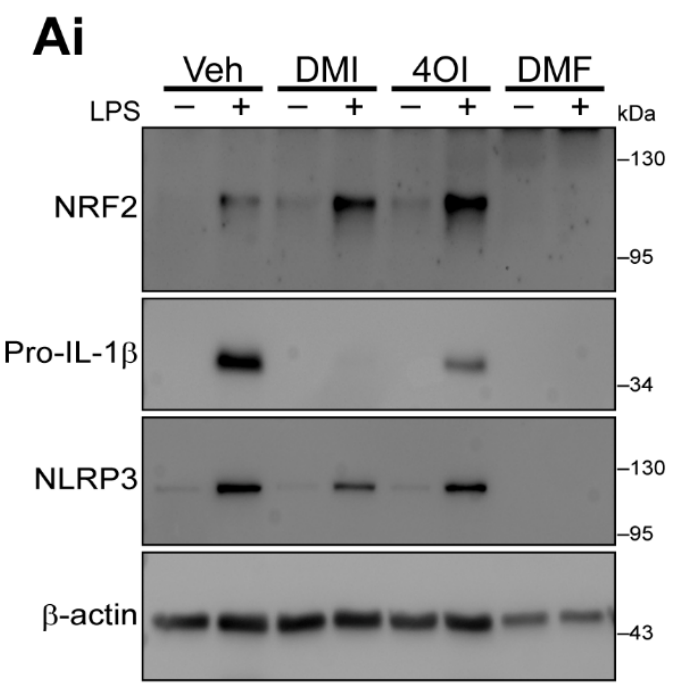

$\mathrm{Bi}$

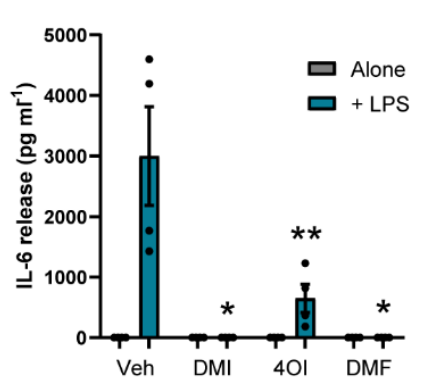

$\mathbf{C i}$

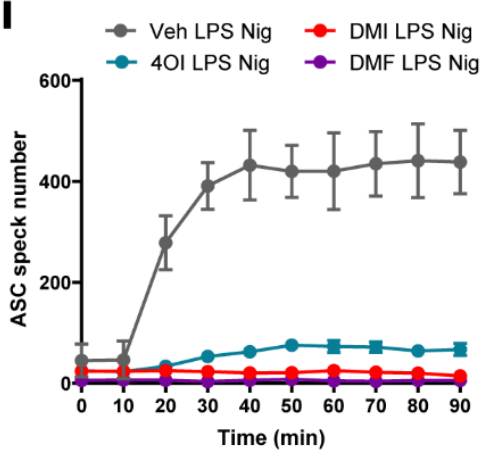

Cii

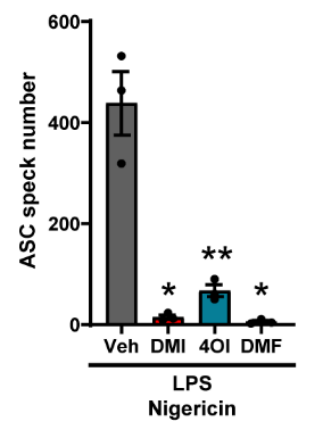

$\mathrm{Bii}$

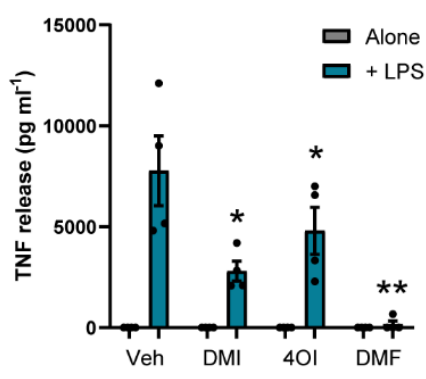

Ciii
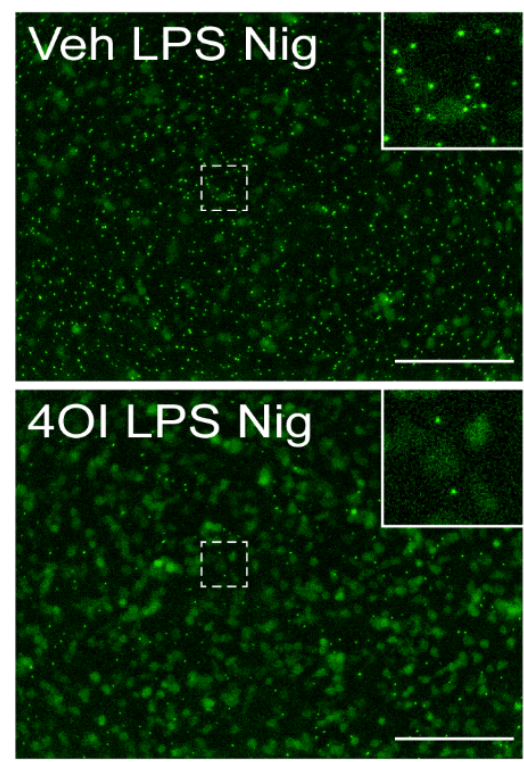
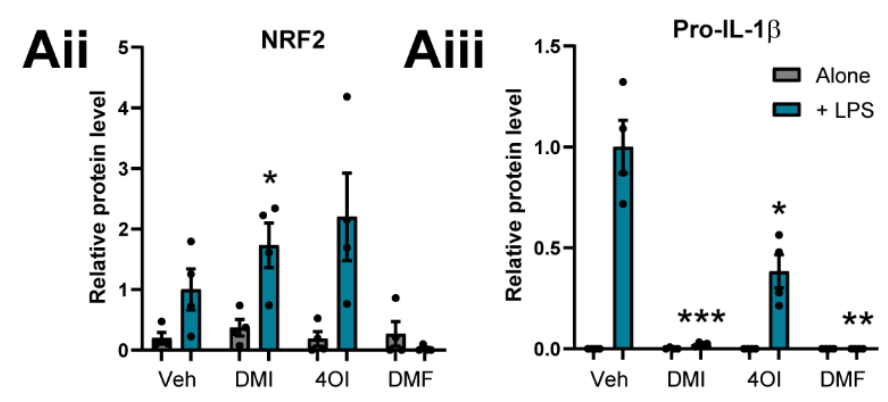

NLRP3

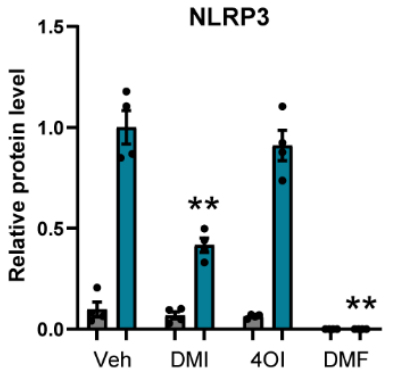

Biii
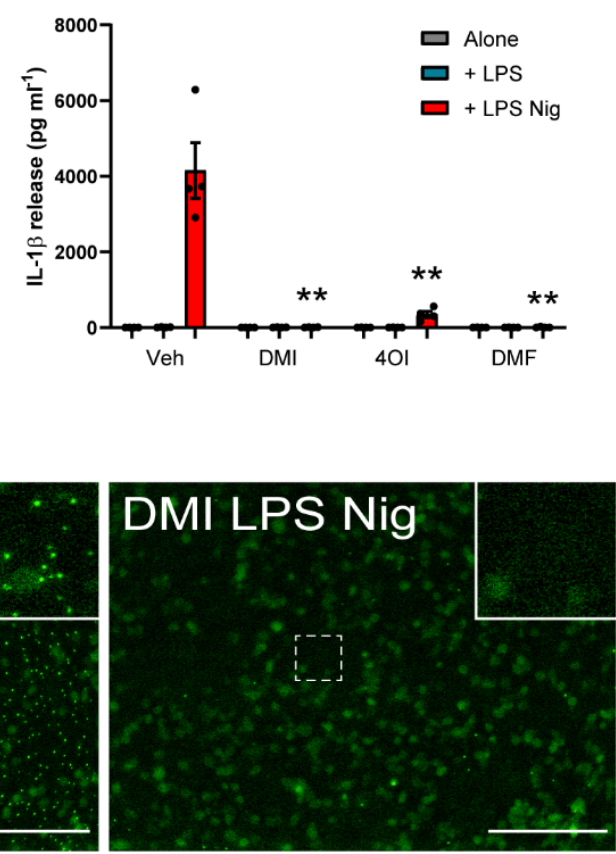

DMF LPS Nig

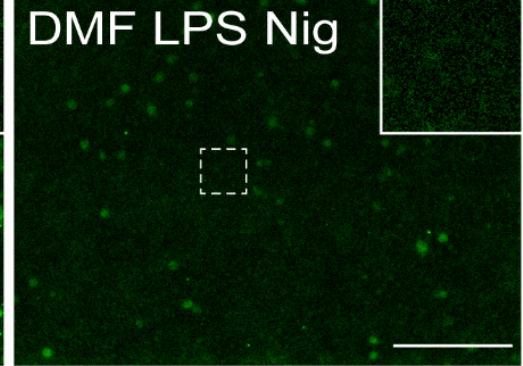


144 Figure 1. Pre-treatment with itaconate and fumarate derivatives reduces NLRP3 priming and 145 canonical inflammasome activation. (A) WT BMDMs were treated with vehicle (DMSO), DMI, 4OI 146 or DMF $(125 \mu \mathrm{M}, 20 \mathrm{~h})$. LPS $\left(1 \mu \mathrm{g} \mathrm{ml}^{-1}, 4 \mathrm{~h}\right)$ was then added to the wells to induce priming $(\mathrm{n}=4)$. 147 (Ai) Cell lysates were probed by western blotting for NRF2, pro-IL-1 $\beta$ and NLRP3 protein, and (Aii148 iv) densitometry was performed on each independent experiment (expressed relative to Veh+LPS 149 treatment). (B) WT BMDMs were treated as above, followed by nigericin (10 $\mu \mathrm{M}, 60 \mathrm{~min}$; $\mathrm{n}=4)$. 150 Supernatants were assessed for (Bi) IL-6, (Bii) TNF and (Biii) IL-1 $\beta$ content by ELISA. (C) ASC151 citrine BMDMs were treated as above, followed by nigericin $(10 \mu \mathrm{M}, 90 \mathrm{~min} ; \mathrm{n}=3)$. ASC speck 152 formation was measured over a period of $90 \mathrm{~min}$. Image acquisition began immediately after addition 153 of nigericin. (Ci) ASC speck number per field of view was quantified over 90 min. (Cii) ASC speck 154 number and (Ciii) fluorescence images after $90 \mathrm{~min}$ nigericin treatment are shown. Scale bars are 200 $\mu \mathrm{m}$. Data are presented as mean \pm SEM. Data were analysed using repeated-measures one-way (Cii) or two-way (Aii-iv, B) ANOVA with Dunnett's post-hoc test (versus Veh treatment within each group). $* \mathrm{P}<0.05 ; * * \mathrm{P}<0.01, * * * \mathrm{P}<0.001$.

\section{Itaconate and fumarate derivatives directly inhibit the NLRP3 activation step}

159 To determine whether NLRP3 inflammasome inhibition was a direct effect of the itaconate derivatives, 160 LPS-primed WT BMDMs were treated with DMI, 4OI and DMF prior to nigericin stimulation, and 161 this resulted in inhibition of IL-1 $\beta$ release, as well as small reductions in cell death (Figure 2Ai, Aii). 162 Western blotting of the cell lysates demonstrated that the levels of pro-IL-1 $\beta$ were consistent between treatments, confirming that in this protocol expression of pro-IL-1 $\beta$ was unaffected by DMI, $4 \mathrm{OI}$ or DMF (Figure 2Aiii, Supplementary Figure 2A). Dose-dependent inhibition of IL-1 $\beta$ release was observed for each treatment, with minimal reductions in cell death (Supplementary Figure 3). ASC speck formation was measured in LPS-primed ASC-citrine BMDMs treated with DMI, 4OI and DMF prior to nigericin. Each of these metabolite derivatives inhibited ASC speck formation in response to nigericin, suggesting that these compounds were also able to directly block NLRP3 inflammasome activation independently of their effects on the priming response (Figure 2Bi-iii). Fluorescence and bright-field images are shown (Figure 2Bi, Supplementary Figure 4). DMF treatment after LPS priming did not induce morphologically observable cell death, indicating that DMF was able to inhibit NLRP3 inflammasome activation independent of toxicity at this concentration and duration of incubation, and

173 this has been reported previously (Garstkiewicz et al., 2017; Liu et al., 2016; Miglio et al., 2015)

174 (Supplementary Figure 3). LPS-primed primary BMDMs treated with DMI, 4OI or DMF and 175 subsequent nigericin stimulation were lysed directly in-well without removing the supernatant, and 176 western blotting confirmed reductions in caspase-1 activation, and gasdermin D and IL-1 $\beta$ cleavage 177 (Figure 2C, Supplementary Figure 2B). NRF2 levels were increased by DMI, 4OI and DMF treatment 178 after LPS priming, although this was not observed in cells that received subsequent nigericin 
179 stimulation (Figure 2C, Supplementary Figure 2B), and itaconate-mediated NLRP3 inhibition is 180 suggested to be independent of NRF2 (Hooftman et al., 2020; Swain et al., 2020). MMF treatment 181 limited NLRP3 activation in LPS-primed BMDMs, although it was not as potent as DMF (Fig 2Di, 182 Dii). We confirmed that exogenous itaconate treatment also inhibited NLRP3 activation in LPS-primed 183 BMDMs, although much higher doses were required because it is less cell permeable (Supplementary 184 Figure 5) (Swain et al., 2020). To determine whether the inhibitory effects of itaconate and fumarate 185 derivatives were relevant in human macrophages, LPS-primed human MDMs were treated with DMI, $1864 \mathrm{OI}$ and DMF prior to nigericin stimulation. 4OI and DMF alone appeared to induce IL-1 $\beta$ release, 187 although slight increases in cell death were observed for these treatments, which may have allowed 188 passive release of unprocessed pro-IL-1 $\beta$ (Figure 2Ei). Both DMI and DMF reduced nigericin-induced

189 IL-1 $\beta$ release, whereas 4OI did not significantly reduce IL-1 $\beta$ release at this dose (Figure 2Ei). No 190 inhibition of cell death was observed (Figure 2Eii). Thus, the itaconate and fumarate derivatives were 191 able to directly inhibit NLRP3 activation in peripheral macrophages, independent of their effects on 192 priming. 
$\mathbf{A i}$

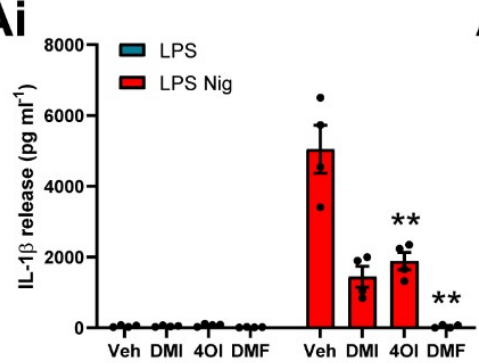

Bi

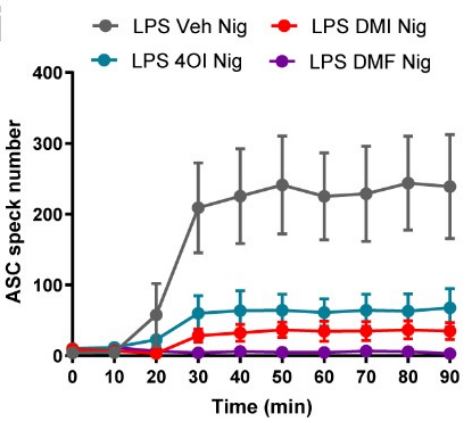

$\mathrm{Bii}$

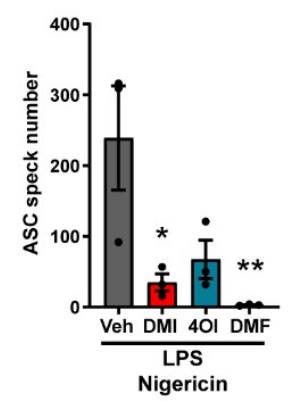

C

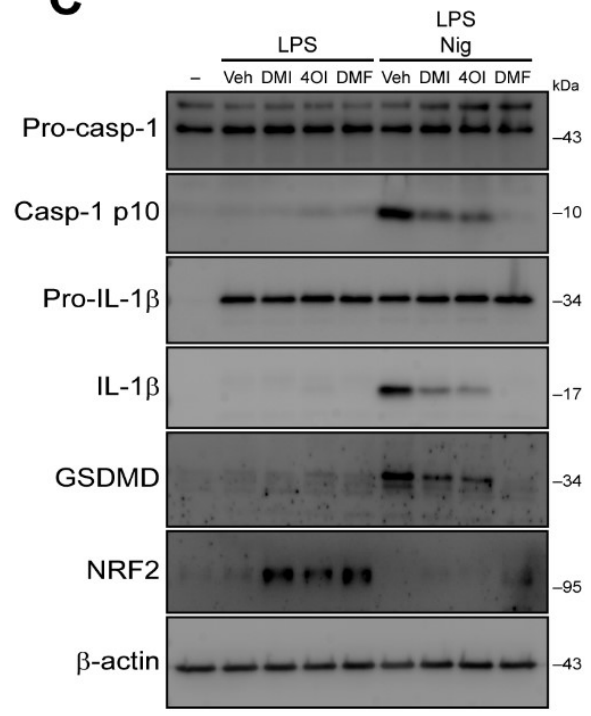

Aii

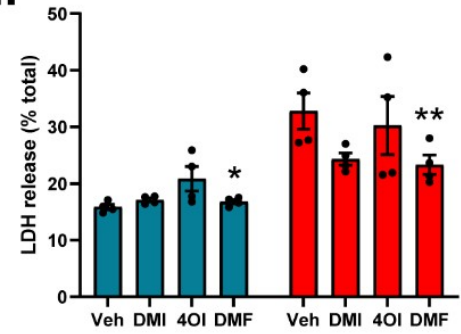

\section{Biii}
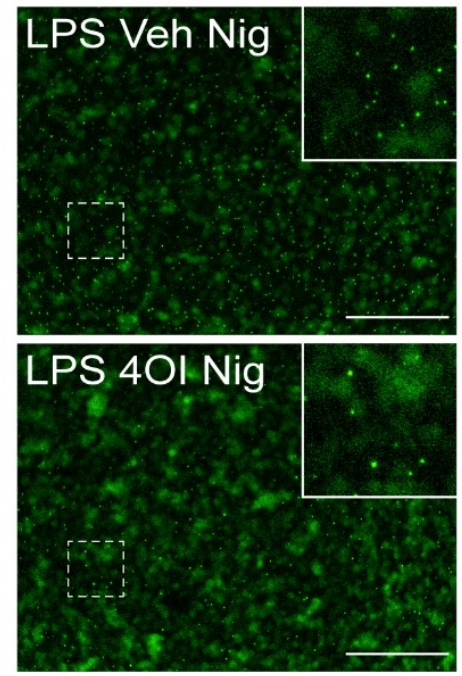

Di

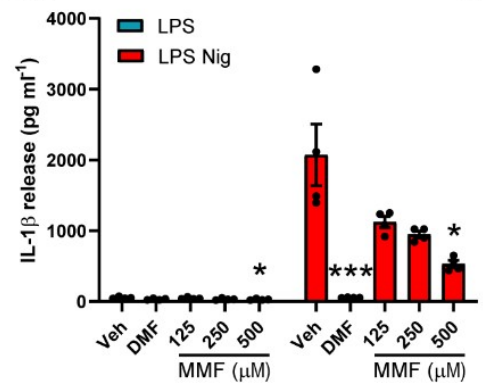

Dii

Ei

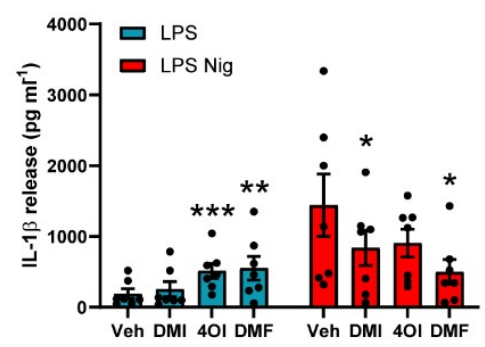

Eii
Aiii

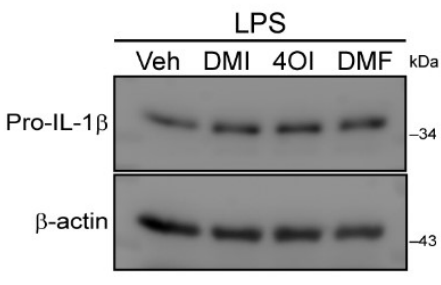

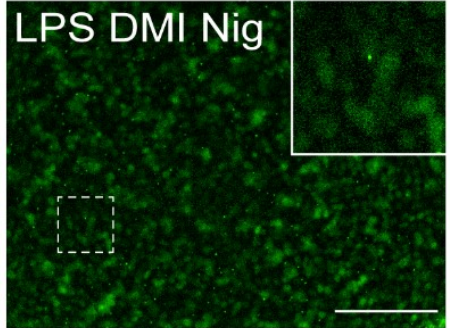
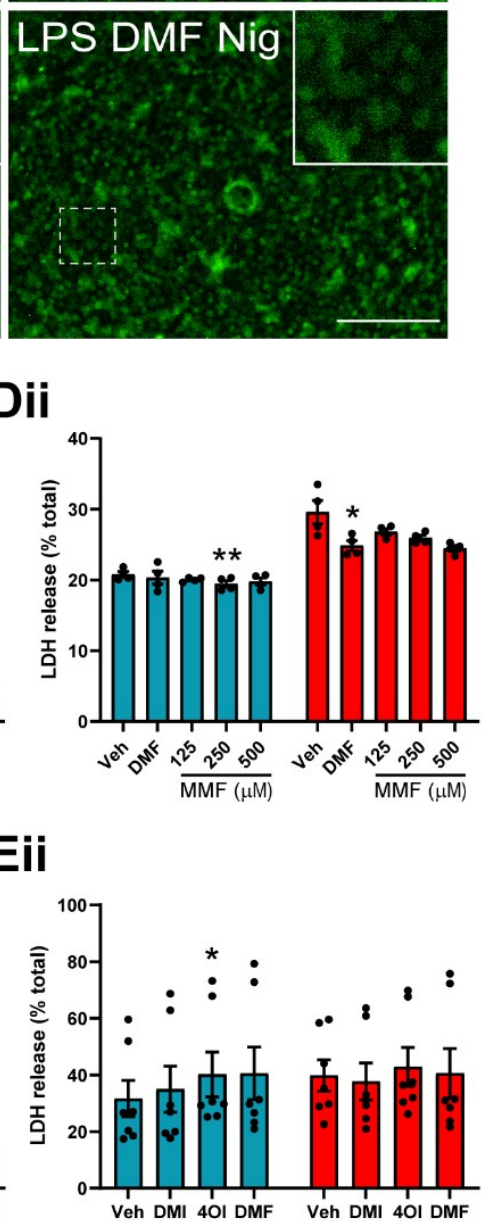

194 Figure 2. Itaconate and fumarate derivatives directly inhibit the NLRP3 activation step in murine and human macrophages. (A) WT BMDMs were primed with LPS $\left(1 \mu \mathrm{g} \mathrm{ml}{ }^{-1}, 4 \mathrm{~h}\right)$ before treatment with vehicle (DMSO), DMI, 4OI or DMF (125 $\mu \mathrm{M}, 15 \mathrm{~min}$ ). Nigericin was then added to 
the well $(10 \mu \mathrm{M}, 60 \mathrm{~min} ; \mathrm{n}=4)$. Supernatants were assessed for (Ai) IL-1 $\beta$ release and (Aii) cell death (LDH release). (Aiii) Cell lysates were probed by western blotting for pro-IL-1 $\beta$ protein. See Supplementary Figure 2A. (B) ASC-citrine BMDMs were treated as above, and ASC speck formation was measured over a period of $90 \mathrm{~min}(\mathrm{n}=3)$. Image acquisition began immediately after addition of nigericin. (Bi) ASC speck number per field of view was quantified over 90 min. (Bii) ASC speck number and (Biii) fluorescence images after 90 min nigericin treatment are shown. Scale bars are 200 $\mu \mathrm{m}$. (C) WT BMDMs were treated as above and then lysed in-well and probed for several markers of inflammasome activation by western blotting $(n=4)$. See Supplementary Figure 2B. (D) WT BMDMs were LPS primed $\left(1 \mu \mathrm{g} \mathrm{ml}^{-1}, 4 \mathrm{~h}\right)$ before treatment with vehicle (DMSO), DMF $(125 \mu \mathrm{M})$ or MMF (125-500 $\mu \mathrm{M}, 15 \mathrm{~min})$. Nigericin was then added to the well $(10 \mu \mathrm{M}, 60 \mathrm{~min} ; \mathrm{n}=4)$. Supernatants were assessed for (Di) IL-1 $\beta$ release and (Dii) LDH release. (E) Human MDMs were LPS primed $\left(1 \mu \mathrm{g} \mathrm{ml} \mathrm{m}^{-}\right.$ 1, $4 \mathrm{~h}$ ) before treatment with vehicle (DMSO), DMI, 4OI or DMF (125 $\mu \mathrm{M}, 15 \mathrm{~min})$. Nigericin was then added to the well $(10 \mu \mathrm{M}, 60 \mathrm{~min}$; $\mathrm{n}=7)$. Supernatants were assessed for (Ei) IL-1 $\beta$ release and (Eii) LDH release. Supernatants were assessed for cytokine content by ELISA. Data are presented as mean \pm SEM. Data were analysed using repeated-measures one-way (Bii) or two-way (Ai, Aii, D, E) ANOVA with Dunnett's post-hoc test (versus Veh treatment within each group). ${ }^{*} \mathrm{P}<0.05 ; * * \mathrm{P}<0.01$; $* * * \mathrm{P}<0.001$.

Itaconate and fumarate derivatives inhibit NLRP3 priming and canonical activation in mixed glia and OHSCs

Despite accumulating evidence in peripheral immune cells, little is known about the importance of immunometabolic regulation of inflammasome priming and activation in the brain, where microglia are thought to be the predominant source of inflammasomes (Sheppard et al., 2019). Thus, we assessed the effect of itaconate derivative pre-treatment on mixed glial cultures, which consist of approximately $80 \%$ astrocytes, $10 \%$ microglia and 10\% oligodendrocyte/type-2 astrocyte progenitor cells (Pinteaux et al., 2002), and organotypic hippocampal slice cultures (OHSCs), which we recently validated as a model for studying microglial NLRP3 responses (Hoyle et al., 2020). DMF was not included in these pre-treatment experiments due to its toxicity observed in murine BMDMs (Figure 1, Supplementary Figure 1), although it has been previously demonstrated to inhibit IL-1 $\beta$, IL-6, and to a lesser extent TNF expression at a lower dose in rat neonatal microglial cultures (Wilms et al., 2010). DMI and 4OI alone did not induce detectable NRF2 accumulation in mixed glial cultures (Figure 3Ai, Aii). DMI treatment did not significantly enhance LPS-induced NRF2 accumulation, although increases were observed; similarly, 4OI did not increase LPS-induced NRF2 levels (Figure 3Ai, Aii). IкBל protein levels were not measured. Despite this, both DMI and 4OI reduced pro-IL-1 $\beta$ production in mixed glial cultures, without affecting NLRP3 protein levels (Figure $3 \mathrm{Ai}-\mathrm{iv}$ ). IL-1 $\beta$ release upon subsequent 
232 (Figure 3Av, Avi). Given that the inhibition of pro-IL-1 $\beta$ production and mature IL-1 $\beta$ release was 233 comparable between DMI and 4OI in mixed glia, only 4OI was used in OHSCs. NRF2 accumulation 234 could not be reliably detected in OHSCs upon LPS priming (data not shown). 4OI reduced the 235 production of pro-IL-1 $\beta$ in response to LPS priming, but did not affect NLRP3 production (Figure $3 \mathrm{Bi}-$ 236 iii). 4OI pre-treatment strongly inhibited IL-1 $\beta$ release in response to LPS and nigericin treatment and 237 reduced cell death (Figure 3Biv, Bv). These data suggested that itaconate derivatives were able to limit 238 the priming of inflammasome responses, and that this may be a relevant mechanism to regulate 239 microglial inflammatory gene expression. 


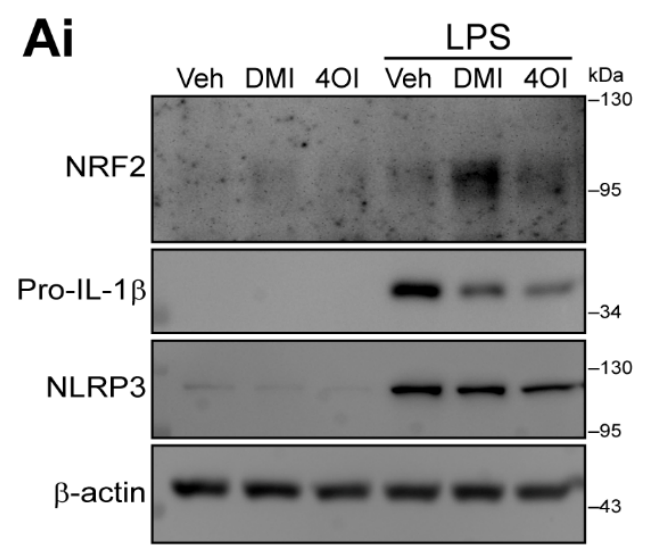

\section{Aiv}

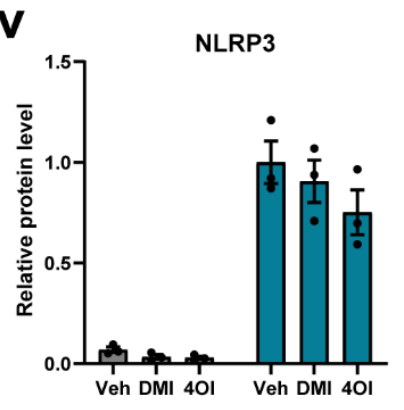

Aii

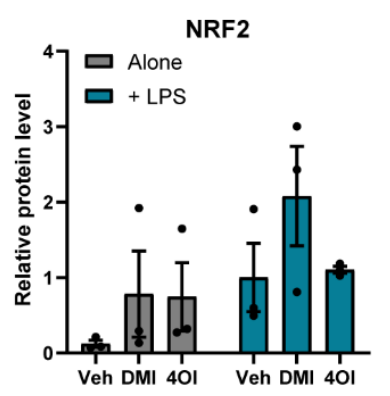

Aiii

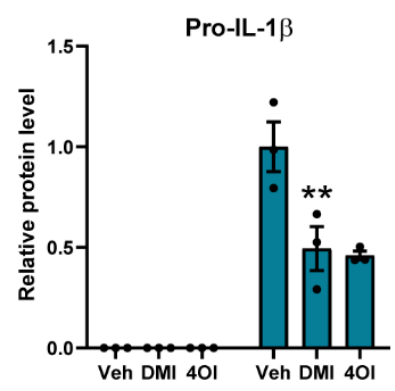

Avi
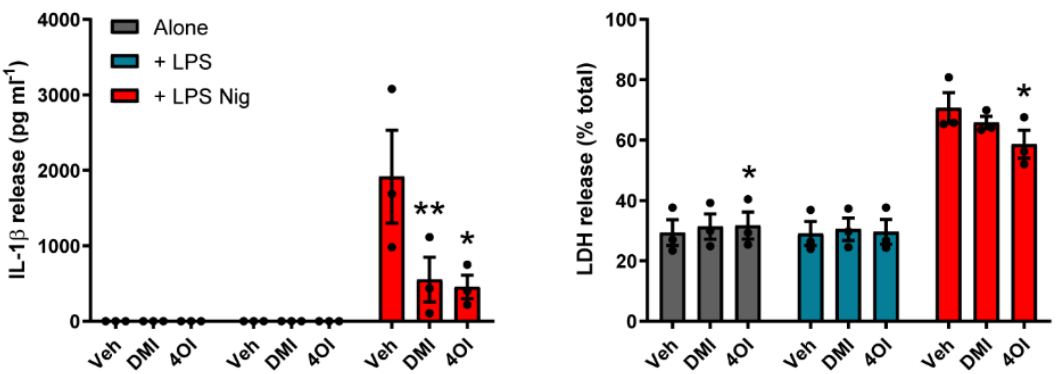

$\mathrm{Bi}$

$\mathrm{Bii}$

Biii

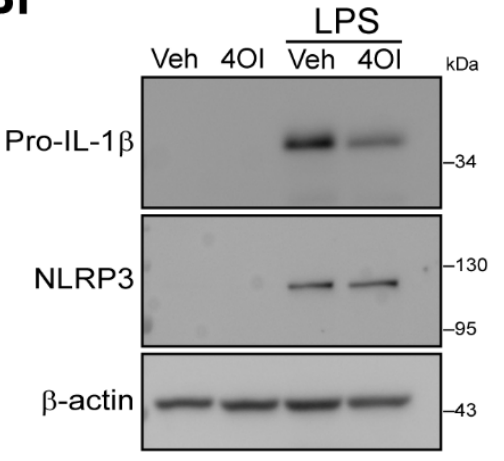

Biv

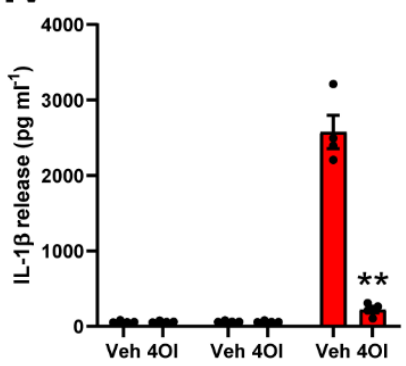

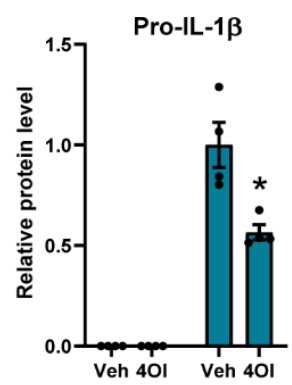

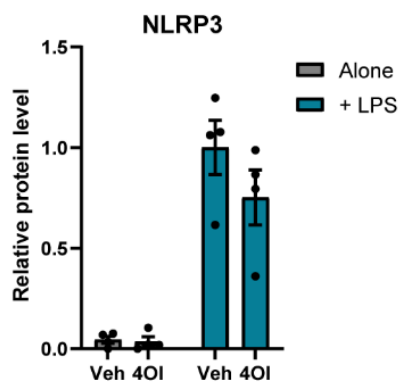

Bv

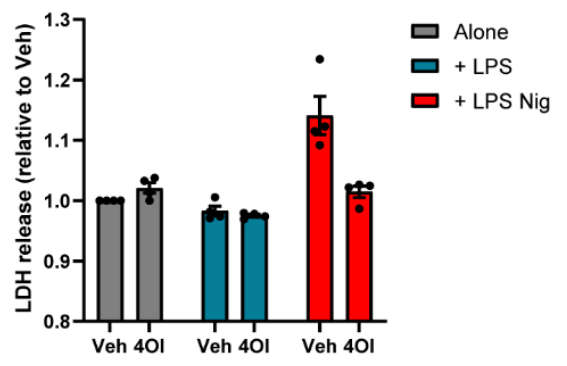

Figure 3. Itaconate and fumarate derivative pre-treatment inhibits NLRP3 priming in mixed glia and OHSCs. (A) WT mixed glia were treated with vehicle (DMSO), DMI or 4OI (125 $\mu \mathrm{M}, 21 \mathrm{~h}$ ). LPS $\left(1 \mu \mathrm{g} \mathrm{ml}^{-1}, 3 \mathrm{~h}\right.$ ) was then added to the wells to induce priming $(\mathrm{n}=3)$. (Ai) Cell lysates were probed by 
245 on each independent experiment (expressed relative to Veh + LPS treatment). (Av, Avi) WT mixed 246 glia were treated as above, followed by nigericin $(10 \mu \mathrm{M}, 60 \mathrm{~min} ; \mathrm{n}=3)$. Supernatants were assessed

247 for (Av) IL-1 $\beta$ release and (Avi) cell death (LDH release). (B) WT OHSCs were treated with vehicle 248 (DMSO) or 4OI $(125 \mu \mathrm{M}, 21 \mathrm{~h})$. LPS $\left(1 \mu \mathrm{g} \mathrm{ml} \mathrm{m}^{-1}, 3 \mathrm{~h}\right)$ was then added to the wells to induce priming $249 \quad(n=4)$. (Bi) OHSC lysates were probed by western blotting for pro-IL-1 $\beta$ and NLRP3 protein, and (Bii250 iiii) densitometry was performed on each independent experiment (expressed relative to Veh+LPS 251 treatment). (Biv, Bv) WT OHSCs were treated as above, followed by nigericin (10 $\mu \mathrm{M}, 90 \mathrm{~min} ; \mathrm{n}=4)$. 252 Supernatants were assessed for (Biv) IL-1 $\beta$ release and (Bv) LDH release. Supernatants were assessed 253 for cytokine content by ELISA. Data are presented as mean $\pm \mathrm{SEM}$. Data were analysed using repeated254 measures two-way ANOVA with Dunnett's (A) or Sidak's (B) post-hoc test (versus Veh treatment 255 within each group). $* \mathrm{P}<0.05 ; * * \mathrm{P}<0.01 ; * * * \mathrm{P}<0.001$.

256 Given that 4OI appeared to inhibit mature IL-1 $\beta$ release more strongly than it inhibited pro-IL-1 $\beta$ 257 production in OHSCs, we investigated whether itaconate and fumarate derivatives could directly limit 258 NLRP3 inflammasome activation in mixed glia and OHSCs. LPS-primed mixed glial cultures were 259 treated with DMI and 4OI prior to nigericin stimulation, and this reduced IL-1 $\beta$ release but did not 260 inhibit cell death (Figure 4Ai, Aii). Similarly, LPS-primed OHSCs were treated with DMI, 4OI, DMF 261 and MMF prior to nigericin stimulation. The compounds alone did not exhibit any toxicity, although a 262 small increase in cell death was observed in response to DMF treatment, nor did they induce ASC 263 speck formation (Supplementary Figure 6). Following nigericin stimulation, 4OI and DMF 264 significantly inhibited IL-1 $\beta$ release, though this was not accompanied by significant reductions in 265 ASC speck formation (Figure 4Bi, Bii). Only MMF treatment significantly reduced nigericin-induced 266 cell death, although this reduction was modest (Figure 4Biii). Representative immunofluorescence 267 images are shown (Figure 4Biv). Together, these data suggested that the itaconate and fumarate 268 derivatives could reduce microglial NLRP3 responses. 
$\mathbf{A i}$

Aii
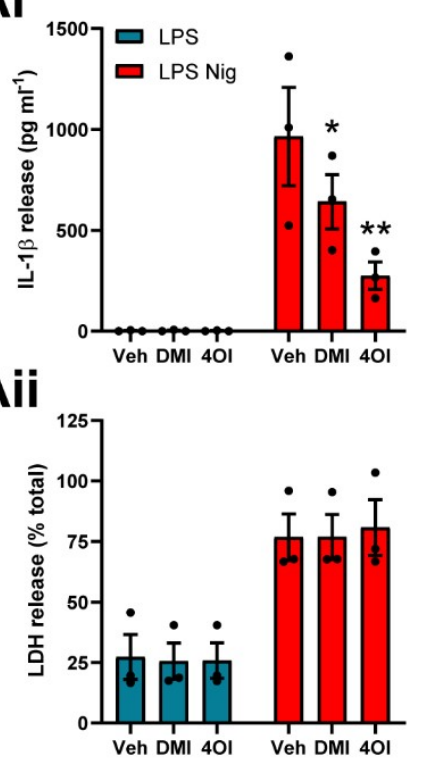

$\mathrm{Bi}$

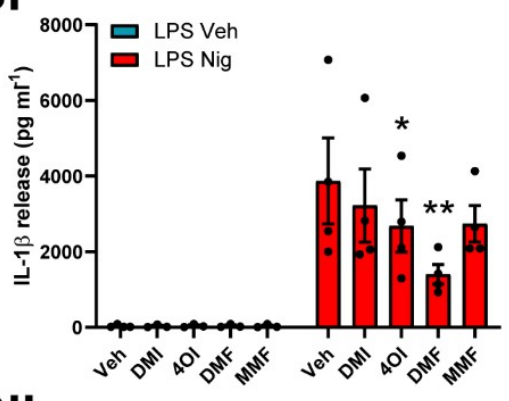

$\mathrm{Bii}$

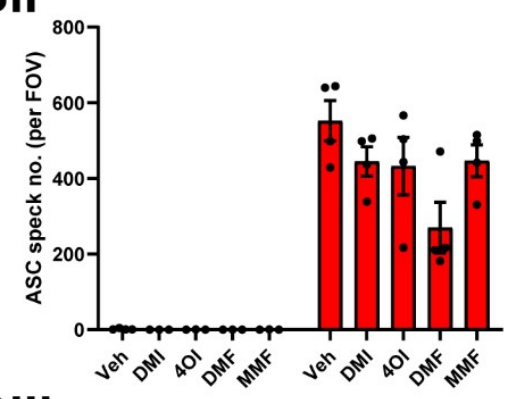

Biii

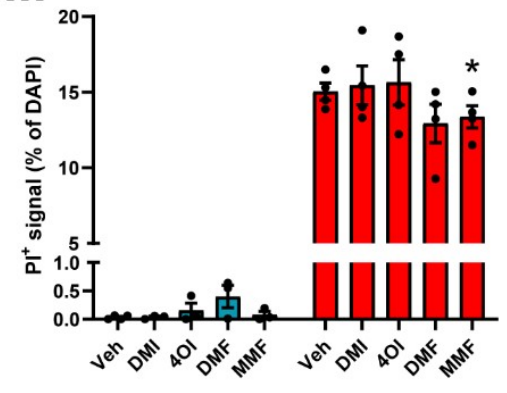

Biv
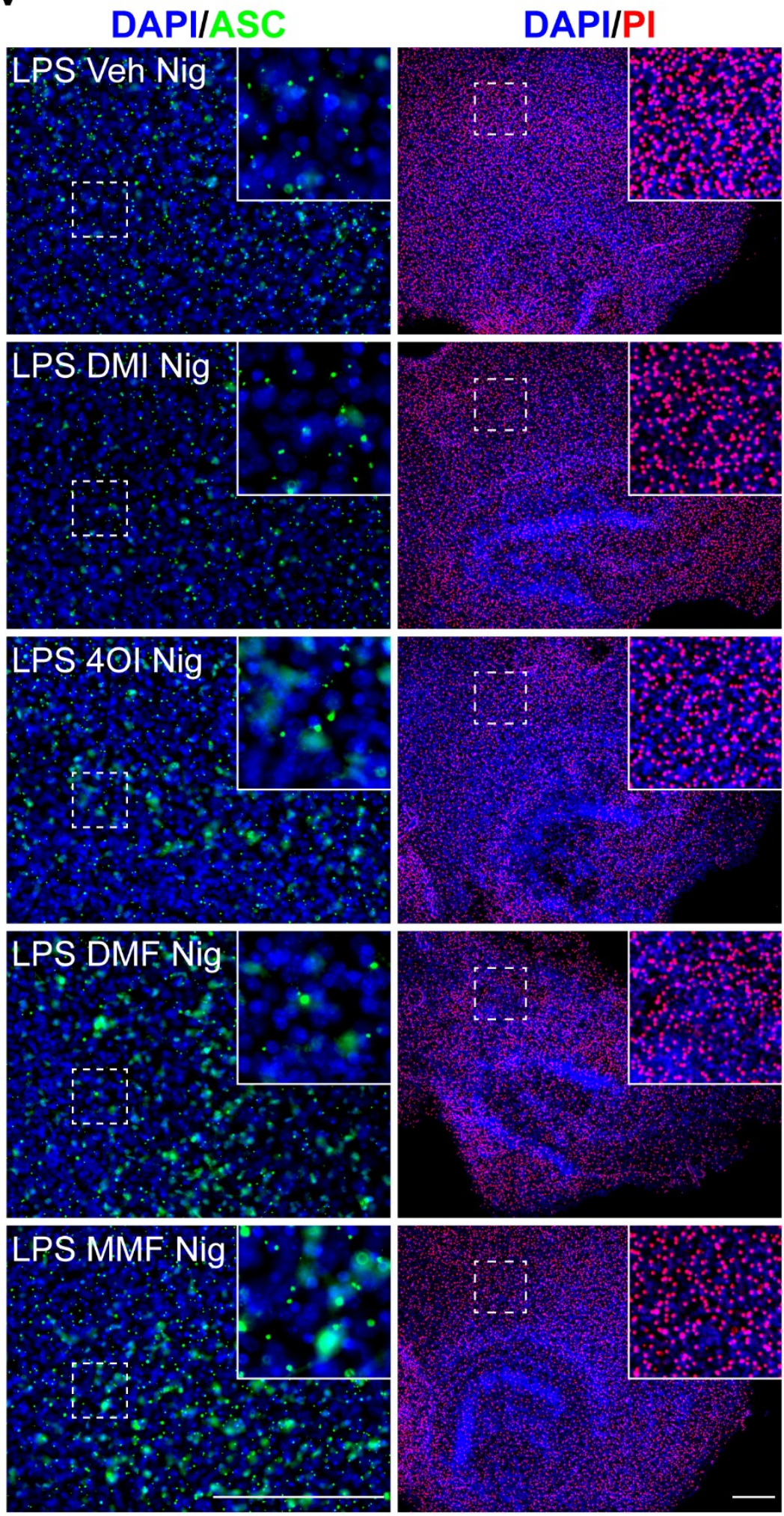

270 Figure 4. Itaconate and fumarate derivatives partly inhibit canonical NLRP3 activation in LPS-

271 primed mixed glia and OHSCs. (A) Mixed glia were primed with LPS $\left(1 \mu \mathrm{g} \mathrm{m}{ }^{-1}, 3 \mathrm{~h}\right)$ before 
treatment with vehicle, DMI or $4 \mathrm{OI}(125 \mu \mathrm{M}, 15 \mathrm{~min})$. Nigericin was then added to the well $(10 \mu \mathrm{M}$, 60 min; $n=3$ ). Supernatants were assessed for (Ai) IL-1 $\beta$ release and (Aii) cell death (LDH release). (B) WT OHSCs were primed with LPS $\left(1 \mu \mathrm{g} \mathrm{ml}^{-1}, 3 \mathrm{~h}\right)$ before treatment with vehicle (DMSO), DMI, 4OI, DMF $(125 \mu \mathrm{M})$ or MMF (500 $\mu \mathrm{M}, 15 \mathrm{~min})$. Vehicle (ethanol) or nigericin was then added to the well (10 $\mu \mathrm{M}, 90 \mathrm{~min} ; \mathrm{n}=3-4)$. Propodium iodide (PI; red, $25 \mu \mathrm{g} \mathrm{ml}^{-1}$ ) was added for the final $30 \mathrm{~min}$ of nigericin treatment. (Bi) Supernatants were assessed for IL-1 $\beta$ content. OHSCs were probed for nuclei (DAPI, blue) and ASC (green) by immunofluorescence staining. (Bii) ASC speck number per field of view was quantified. (Biii) The area of PI-positive staining was determined and is expressed as a \% of total area of DAPI staining. (Biv) Representative images are shown. Images were acquired using widefield microscopy at 20X (ASC) and 5X (PI) magnification. Scale bars are $200 \mu \mathrm{m}$. Data are presented as mean \pm SEM. Data were analysed using repeated-measures two-way ANOVA (A) or mixed effects model (B) with Dunnett's post-hoc test (versus Veh treatment within each group). $* \mathrm{P}<0.05 ; * * \mathrm{P}<0.01$.

LPC (also known as lysolecithin) is a lipid biomolecule that is generated from the cleavage of et al., 2019). LPC levels are regulated by the enzyme lysophosphatidylcholine acyltransferase, which converts LPC back to phosphatidylcholine (Law et al., 2019). Despite its presence during normal physiology, LPC is able to induce demyelination in experimental models of multiple sclerosis (Hall, 1972; Lassmann and Bradl, 2017; Plemel et al., 2018), implicating endogenous LPC dysregulation as a potential factor in multiple sclerosis pathology, although evidence of phospholipase $\mathrm{A}_{2}$ involvement in multiple sclerosis patients is unclear (Trotter et al., 2019). LPC can also activate the NLRP3 and NLRC4 inflammasomes in macrophages, microglia and astrocytes (Freeman et al., 2017), and NLRP3 is reported to be detrimental in experimental autoimmune encephalomyelitis (Coll et al., 2015; Gris et al., 2010; Jha et al., 2010). Thus, LPC-induced NLRP3 inflammasome activation may directly influence LPC-induced demyelination. We assessed whether the itaconate and fumarate derivatives could inhibit NLRP3 activation driven by LPC stimulation in peripheral macrophages. LPS-primed BMDMs were treated with DMI, 4OI, DMF or MCC950, a selective NLRP3 inhibitor (Coll et al., 2015), prior to LPC stimulation. Each of the derivatives inhibited IL-1 $\beta$ release to the same extent as MCC950, indicating inhibition of NLRP3 activation, although no reductions in cell death were observed by any treatment (Figure 5Ai, Aii). Similarly, treatment with MMF inhibited IL-1 $\beta$ release induced by LPC to the same extent as MCC950 (Figure 5Bi, Bii). These data suggest that itaconate and fumarate derivatives could limit NLRP3 activation in peripheral macrophages in response to LPC stimulation. 
Ai

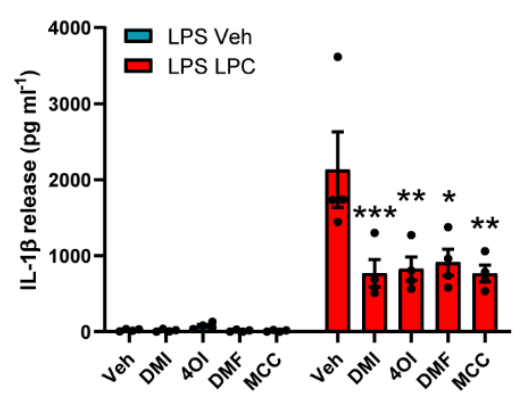

Bi

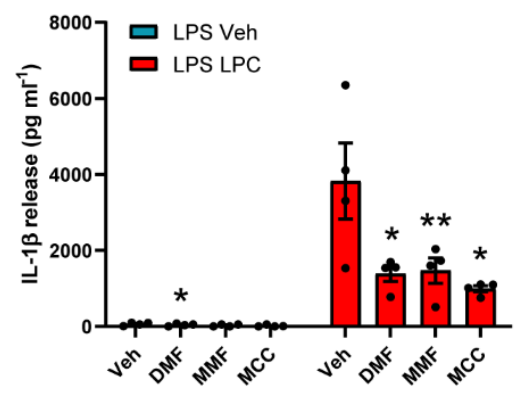

Aii

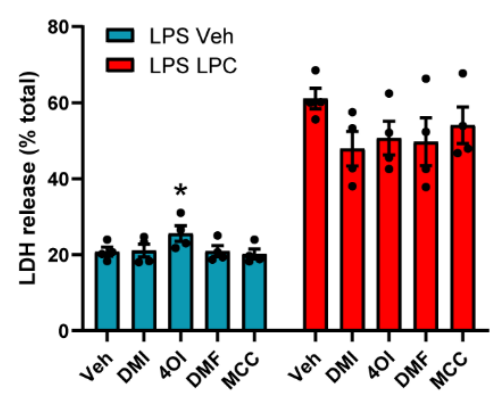

$\mathrm{Bii}$

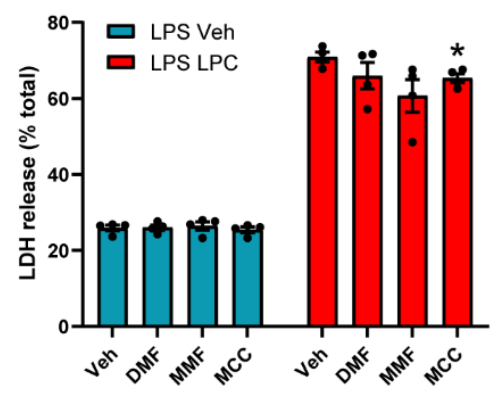

307 Figure 5. Itaconate and fumarate derivatives inhibit NLRP3 activation in response to LPC

308 stimulation. (A) WT BMDMs were primed with LPS $\left(1 \mu \mathrm{g} \mathrm{m}^{-1}, 4 \mathrm{~h}\right)$ before treatment with vehicle 309 (DMSO), DMI, 4OI, DMF $(125 \mu \mathrm{M})$ or MCC950 $(10 \mu \mathrm{M}, 15 \mathrm{~min})$. Vehicle (ethanol) or LPC (100 $310 \mu \mathrm{M}, 60 \mathrm{~min})$ was then added to the well $(\mathrm{n}=4)$. (B) WT BMDMs were LPS primed $\left(1 \mu \mathrm{g} \mathrm{m}^{-1}, 4 \mathrm{~h}\right)$ 311 before treatment with vehicle (DMSO), DMF $(125 \mu \mathrm{M})$, MMF $(500 \mu \mathrm{M})$ or MCC950 $(10 \mu \mathrm{M}$, $31215 \mathrm{~min})$. Vehicle (ethanol) or LPC $(100 \mu \mathrm{M}, 60 \mathrm{~min})$ was then added to the well $(\mathrm{n}=4)$. Supernatants 313 were assessed for (Ai, Bi) IL-1 $\beta$ release by ELISA and (Aii, Bii) cell death (LDH release). Data are 314 presented as mean \pm SEM. Data were analysed using repeated-measures two-way ANOVA with 315 Dunnett's post-hoc test (versus Veh treatment within each group). ${ }^{*} \mathrm{P}<0.05 ; * * \mathrm{P}<0.01 ; * * * \mathrm{P}<0.001$. 


\section{Discussion}

318 Macrophage metabolism has emerged as an important regulator of inflammatory responses (O'Neill

319 and Artyomov, 2019). In particular, exogenous treatment with derivatives of the metabolite itaconate

320 inhibits the expression of NF- $\mathrm{BB}$ secondary response genes such as IL-1 $\beta$ and IL-6 through NRF2

321 accumulation (Mills et al., 2018) and inhibition of $\mathrm{I} \kappa \mathrm{B} \zeta$ translation (Bambouskova et al., 2018).

322 However, the direct effect of itaconate derivative treatment on NLRP3 inflammasome activity is

323 unclear, with a few recent studies indicating inhibition of IL-1 $\beta$ release independently of reductions in

324 pro-IL-1 $\beta$ (Hooftman et al., 2020; Swain et al., 2020). We confirmed that DMI and 4OI are able to

325 inhibit the expression of pro-inflammatory genes in macrophages upon LPS priming and show that this

326 mechanism may have relevance in microglia. We also found that DMI, 4OI, DMF and MMF can

327 directly inhibit biochemical hallmarks of NLRP3 inflammasome activation independently of their

328 effects on inflammasome priming, including in human macrophages. These findings highlight that

329 itaconate and fumarate derivatives could potentially be manipulated therapeutically in NLRP3-driven

330 diseases, conferring the dual benefit of targeting both priming and activation of NLRP3.

331 Exogenous itaconate has different properties from commonly used itaconate derivatives such as DMI

332 and 4OI, which exhibit greater electrophilicity (Swain et al., 2020). For example, exogenous itaconate

333 does not appear to strongly drive NRF2 signalling, nor does it inhibit $\mathrm{I} \kappa \mathrm{B} \zeta$ translation, and so any

334 effects of itaconate derivatives, although still relevant, may not directly reflect the physiology of

335 endogenous itaconate (Swain et al., 2020). It has also been shown that DMI is not metabolised to

336 itaconate in the cytosol (ElAzzouny et al., 2017). Nevertheless, whilst itaconate derivatives may not be

337 appropriate to simulate the actions of endogenous itaconate, any therapeutic potential for these

338 compounds maintains importance. Interestingly, Swain et al. (2020) also suggested that itaconate

339 derivatives, including exogenous itaconate, can inhibit NLRP3 activation independently of effects on

340 NRF2 and priming. However, itaconate treatments were only applied prior to LPS priming, instead of

341 treating LPS-primed BMDMs with itaconate prior to NLRP3 activation. Thus, it remains possible that

342 other effects of itaconate pre-treatment, such as reduction in NLRP3 protein levels during LPS priming,

343 could have limited IL-1 $\beta$ release. We have addressed this in the current study, complementing

344 observations that 4OI specifically inhibited NLRP3 activation in LPS-primed cells through direct

345 interaction with cysteine 548 on murine NLRP3, preventing NEK7 binding (Hooftman et al., 2020).

346 This mechanism is plausible, given that DMI, 4OI and DMF are electrophiles that modify cysteine

347 residues on target proteins including KEAP1 (Linker et al., 2011; Mills et al., 2018), GAPDH 
348 (Kornberg et al., 2018; Liao et al., 2019), and more than a thousand other proteins in LPS-primed RAW 349 macrophages (Qin et al., 2020).

350 We suggest that the inhibitory mechanisms of itaconate and fumarate derivatives on NLRP3 priming 351 and canonical activation may be consistent in microglia. Thus, the implications of immunometabolic 352 regulation of inflammasome responses in the brain should be explored further, particularly in the 353 context of brain pathology. Transcriptomic databases indicate that microglia exhibit relatively high 354 expression of Nfe2l2 (NRF2) and Keapl (Schaum et al., 2018; Zhang et al., 2014). The itaconate355 synthesising enzyme $\operatorname{Irgl}$ (also known as Acod1) was recently shown to be upregulated in response to 356 LPS treatment in OHSCs (Chausse et al., 2020), driving subsequent itaconate production, and this was 357 prevented by microglial depletion, indicating that this is primarily a microglial response. Furthermore, 358 exogenous treatment with 4OI limited LPS-induced IL-6 production but did not affect TNF (Chausse 359 et al., 2020). While mixed glia and OHSCs are useful tools to study microglial function, further studies 360 are required to fully understand the physiological relevance of microglial metabolic reprogramming in 361 vivo. NRF2 activation is associated with a protective effect in experimental ischaemic stroke models 362 (Liu et al., 2019), and IRG-deficient mice exhibit exacerbated brain damage to acute ischaemic stroke, according to a recent pre-print (Kuo et al., 2020b). These studies suggest that itaconate production 364 could be an endogenous, protective response to limit ischaemic damage. Our previous report showed 365 increased levels of IL-1 $\beta$ and NLRP3 expression after ischaemic stroke, but NLRP3 deficiency or inhibition did not improve stroke outcome (Lemarchand et al., 2019). This could suggest that endogenous itaconate production within the brain in response to ischaemia, whilst too late to inhibit inflammatory cytokine production, may be able to limit NLRP3 inflammasome activation. It is important to note that detection of increased NRF2 levels in response to DMI and 4OI treatment was not reliable in the microglial models employed in this study, either due to a lower amount of NRF2 signalling, questioning the relevance of NRF2 activation in the brain, or due to the instability of NRF2 protein during OHSC sample processing steps such as water-bath sonication. It should also be noted that the extent of NLRP3 inhibition mediated by the itaconate and fumarate derivatives in the OHSCs was lower than in the BMDM and mixed glial assays. It is possible that higher doses or longer treatment

375 times of the metabolite derivatives would result in greater NLRP3 inhibition, given that we have 376 previously shown that MCC950 is able to potently inhibit IL-1 $\beta$ release and ASC speck formation in 377 OHSCs (Hoyle et al., 2020). 
378 We demonstrate that DMF, an approved clinical treatment for relapsing-remitting multiple sclerosis

379 (Fox et al., 2012; Gold et al., 2012b; Linker et al., 2011) and psoriasis (Smith, 2017), is an effective

380 NLRP3 inhibitor in both macrophages and microglia, as has been suggested previously (Garstkiewicz

381 et al., 2017; Liu et al., 2016; Miglio et al., 2015). We also show that DMF, as well as DMI and 4OI,

382 limits NLRP3 activation in response to LPC stimulation of macrophages, a demyelinating agent that

383 can drive NLRP3 and NLRC4 activation in macrophages, microglia and astrocytes (Freeman et al.,

384 2017). The mechanism underlying DMF's beneficial effect in multiple sclerosis and psoriasis is

385 unclear, and is commonly suggested to be mediated via NRF2 activation, although NRF2-independent

386 effects are also reported (Schulze-Topphoff et al., 2016). Given that DMF is able to potently inhibit

387 NLRP3 activation and directly inhibit gasdermin D cleavage (Humphries et al., 2020), and that NLRP3

388 is detrimental in the experimental autoimmune encephalomyelitis model of multiple sclerosis (Coll et

389 al., 2015; Gris et al., 2010; Jha et al., 2010), it is possible that DMF's protective response in multiple sclerosis is in part mediated through dampened microglial and macrophage NLRP3 activation that may promote or result from neuronal demyelination. Inhibition of NLRP3 activation in macrophages or microglia could also facilitate other inflammatory responses that promote clearance of damaged myelin and remyelination (Cunha et al., 2020). Evidence of caspase-1 activation and gasdermin D-mediated

394 pyroptosis has also been observed in the CNS of multiple sclerosis patients and in animal models (Li et al., 2019; McKenzie et al., 2018), further implicating NLRP3 involvement. Upon administration, DMF is hydrolysed to MMF and can be detected in the serum (Gold et al., 2012a; Litjens et al., 2004). MMF also induces KEAP1 cysteine alkylation and NRF2 activation (Linker et al., 2011), suggesting it may exert similar inhibitory effects to DMF on the priming response. Importantly, here we confirm that MMF can inhibit NLRP3 activation in response to both nigericin and LPC stimulation in macrophages, suggesting that NLRP3 inhibition could indeed be a relevant in vivo mechanism for DMF treatment. While DMF exhibited toxic effects during the pre-treatment experiments, as its dose was matched to that of 4OI, it is likely that titration of the DMF concentration would result in reduced toxicity but similar potency. Given that DMI and 4OI, which both also drive NRF2 accumulation, exerted similar inhibitory effects on nigericin- and LPC-induced NLRP3 activation, it is possible that these itaconate derivatives may also offer therapeutic potential in the treatment of multiple sclerosis. Indeed, DMI was recently shown to be protective in a mouse model of multiple sclerosis (Kuo et al., 2020a).

We have revealed a two-pronged immunometabolic mechanism of NLRP3 regulation by limiting both 
410 such as itaconate and fumarate may represent a viable therapeutic strategy in NLRP3-driven diseases,

411 although further work is required to confirm this. Future work should also aim to establish whether

412 itaconate and fumarate derivatives inhibit NLRP3 activation through the same or differing

413 mechanisms, either confirming this as a promising therapeutic target for drug design, or revealing novel

414 targets.

4154 Materials and methods

416 Mice

417 In-house colonies of wild-type (WT) and ASC-citrine (Tzeng et al., 2016) C57BL/6 mice at the 418 University of Manchester were maintained to provide primary cell cultures. Animals were allowed free 419 access to food and water and maintained under temperature-, humidity- and light-controlled conditions.

420 All animal procedures adhered to the UK Animals (Scientific Procedures) Act (1986).

\section{Primary murine BMDM preparation}

422 Primary bone marrow-derived macrophages (BMDMs) were prepared by centrifuging the femurs of 423 3-6-month-old WT or ASC-citrine mice of either sex in an Eppendorf tube containing phosphate424 buffered saline (PBS) at 10,000 $\times \mathrm{g}(10 \mathrm{~s})$. Bone marrow was collected and red blood cells were lysed 425 with ACK lysing buffer (Lonza, LZ10-548E). Cells were passed through a cell strainer (70 $\mu \mathrm{m}$ pore 426 size; Corning, 734-2761), centrifuged at $1500 \times \mathrm{g}(5 \mathrm{~min})$, and BMDMs were generated by 427 resuspending and culturing the cell pellet in 70\% Dulbecco's modified Eagle's medium (DMEM; 428 Sigma, D6429) containing 10\% (v/v) foetal bovine serum (FBS; Thermo, 10500064), 100 $429 \mathrm{U} \mathrm{ml}^{-1}$ penicillin and $100 \mu \mathrm{g} \mathrm{ml}^{-1}$ streptomycin (PenStrep; Thermo, 15070063), and supplemented 430 with $30 \%$ L929 mouse fibroblast-conditioned medium for 7 days. Cells were incubated at $37^{\circ} \mathrm{C}, 90 \%$ 431 humidity and 5\% $\mathrm{CO}_{2}$. Before experiments, BMDMs were seeded overnight at a density of $1 \times$ $43210^{6} \mathrm{ml}^{-1}$.

\section{Human monocyte-derived macrophage preparation}

434 Human monocyte-derived macrophages (MDMs) were prepared from human peripheral blood 435 mononuclear cells (PBMCs) obtained from consenting healthy donors (National Health Service Blood 436 and Transplant, Manchester, UK), with full ethical approval from the University Research Ethics 437 Committee at the University of Manchester (ref 2017-2551-3945). In brief, PBMCs were isolated by 
438 Ficoll separation (Thermo) at $400 \times \mathrm{g}(40 \mathrm{~min}$, room temperature) with zero deceleration. PBMCs were 439 washed three times with sterile MACS buffer $(0.5 \%(\mathrm{w} / \mathrm{v})$ bovine serum albumin (BSA), 2 mM EDTA 440 in PBS) before positive selection of CD14 $4^{+}$monocytes by incubation with magnetic CD14 microbeads 441 (Miltenyi Biotec, 130-050-201) (15 min, $4^{\circ} \mathrm{C}$ ) and elution using LS columns (Miltenyi Biotec, 130442 042-401). CD14 monocytes were differentiated to MDMs by culturing for 7 days (at a concentration 443 of $1 \times 10^{6}$ cells ml $^{-1}$ ) in RPMI 1640 (Sigma, R8758) supplemented with 10\% (v/v) FBS, PenStrep and 444 macrophage colony-stimulating factor (M-CSF, $0.5 \mathrm{ng} \mathrm{ml}^{-1}$; Peprotech, 300-25) at 37 $\mathrm{C}^{\circ}, 90 \%$ humidity 445 and $5 \% \mathrm{CO}_{2}$. On day 3 of differentiation, cells were fed by the addition of fresh media containing M446 CSF $\left(0.5 \mathrm{ng} \mathrm{ml}^{-1}\right)$. Before experiments, MDMs were seeded overnight at a density of $1 \times 10^{6} \mathrm{ml}^{-1}$.

\section{Primary murine mixed glial culture preparation}

448 Murine mixed glial cells were prepared from the brains of 2-4-day-old mice of either sex that were killed by cervical dislocation, as described previously (Hoyle et al., 2020). The brains were isolated, cerebral hemispheres dissected and the meninges removed. The remaining brain tissue was homogenised in DMEM containing 10\% (v/v) FBS and PenStrep via repeated trituration, then centrifuged at $500 \times \mathrm{g}$ for $10 \mathrm{~min}$ and the pellet was resuspended in fresh culture medium before being incubated in a flask at $37^{\circ} \mathrm{C}, 90 \%$ humidity and $5 \% \mathrm{CO}_{2}$. After 5 days, the cells were washed, and fresh medium was applied. The medium was subsequently replaced every 2 days. On day 12 of the culture, the cells were seeded at $2 \times 10^{5}$ cells $\mathrm{ml}^{-1}$ in 24 -well plates and incubated for a further 2 days prior to use.

\section{Organotypic hippocampal slice culture (OHSC) preparation}

Seven-day-old mouse pups of either sex were killed by cervical dislocation and the brains were collected in PBS containing glucose $\left(5 \mathrm{mg} \mathrm{ml}^{-1}\right)$. The hippocampi were dissected and placed on filter paper, and $400 \mu \mathrm{m}$ slices were prepared using a McIlwain tissue chopper (Brinkman Instruments). Hippocampal slices were collected and placed on $0.4 \mu \mathrm{m}$ Millicell culture inserts (Merck Millipore,

462 PICM03050), as described previously by Stoppini et al. (1991). Three hippocampal slices were placed on each insert. Slices were maintained in a humidified incubator with $5 \% \mathrm{CO}_{2}$ at $37^{\circ} \mathrm{C}$ with $1 \mathrm{ml} \mathrm{MEM}$ 464 (Gibco, 31095209) containing 20\% (v/v) horse serum (Sigma, H1138), supplemented with HEPES

465 (30 mM; Fisher, 10397023) and insulin (0.1 $\mathrm{mg} \mathrm{ml}^{-1}$; Gibco, 12585014), pH 7.2-7.3. The culture 466 medium was changed every 2 days and slices were used at day 7. 


\section{Treatment protocols}

468 To assess the effect of itaconate and fumarate derivative pre-treatments on inflammasome priming, cells were first treated with vehicle (DMSO), DMI (Sigma, 592498), 4OI (Cayman Chemical,

470 CAY25374) or DMF (all $125 \mu \mathrm{M}$; Sigma, 242926) for $20 \mathrm{~h}$ (BMDM) or $21 \mathrm{~h}$ (mixed glia and OHSC).

LPS ( $1 \mu \mathrm{g} \mathrm{ml}^{-1}$; Sigma, L2654) was then added to the wells for $4 \mathrm{~h}$ (BMDM) or $3 \mathrm{~h}$ (mixed glia and OHSC) to induce priming, followed by nigericin (10 $\mu \mathrm{M}$; Sigma, N7143) for $60 \mathrm{~min}$ (BMDM and mixed glia) or $90 \mathrm{~min}$ (OHSC) to activate the NLRP3 inflammasome.

To assess the direct effect of itaconate and fumarate derivative treatments on canonical NLRP3 inflammasome activation in macrophages, BMDMs and human MDMs were first primed with LPS $\left(1 \mu \mathrm{g} \mathrm{ml}^{-1}\right)$ for $4 \mathrm{~h}$. The medium was then replaced with serum-free DMEM (BMDM) or RPMI (human MDM) containing vehicle (DMSO), DMI, 4OI, DMF (all $125 \mu \mathrm{M}, 15 \mathrm{~min}$ ), MMF (500 $\mu \mathrm{M}, 15 \mathrm{~min}$ Sigma, 651419), exogenous itaconate (1-7.5 mM, $15 \mathrm{~min}$; Sigma, I29204) or the NLRP3 inhibitor MCC950 (10 $\mu \mathrm{M}, 15 \mathrm{~min}$; Sigma, PZ0280), before nigericin $(10 \mu \mathrm{M}, 60 \mathrm{~min})$ or LPC (100 $\mu \mathrm{M}$ in ethanol, $60 \mathrm{~min}$; Sigma, L4129) was added to the culture medium. To assess the direct effect of itaconate and fumarate derivative treatments on NLRP3 inflammasome activation in mixed glia and OHSCs, cells were first primed with LPS $\left(1 \mu \mathrm{g} \mathrm{ml}^{-1}\right)$ for $3 \mathrm{~h}$. The medium was then replaced with serum-free DMEM (mixed glia) or MEM (OHSC) containing vehicle (DMSO), DMI, 4OI, DMF $(125 \mu \mathrm{M}, 15 \mathrm{~min})$ or $\mathrm{MMF}(500 \mu \mathrm{M}, 15 \mathrm{~min})$, before nigericin $(10 \mu \mathrm{M})$ was added to the culture medium for $60 \mathrm{~min}$ (mixed glia) or $90 \mathrm{~min}$ (OHSC). At the end of the experiments, the supernatants were collected and cell or OHSC lysates prepared for further analysis.

Western blotting

Primary BMDMs, mixed glia and OHSCs were lysed with lysis buffer $(50 \mathrm{mM}$ Tris/HCl, $150 \mathrm{mM}$ $\mathrm{NaCl}$, Triton-X-100 1\% v/v, pH 7.3) containing protease inhibitor cocktail (Merck Millipore, 539131). OHSCs were additionally lysed using repeated trituration and brief water bath sonication. Lysates were then centrifuged for $10 \mathrm{~min}$ at $12,000 \times \mathrm{g}$ at $4^{\circ} \mathrm{C}$. In experiments where cells were lysed in-well to assess total protein content, cells were lysed by adding protease inhibitor cocktail and Triton-X-100 $1 \%(\mathrm{v} / \mathrm{v})$ into the culture medium. In-well lysates were concentrated by mixing with an equal volume of trichloroacetic acid (Fisher, 10391351) and centrifuged for $10 \mathrm{~min}$ at $18,000 \times \mathrm{g}$ at $4^{\circ} \mathrm{C}$. The supernatant was discarded, and the pellet resuspended in acetone $(100 \%)$ before centrifugation for 10 
resuspending in Laemmli buffer (2X). Samples were analysed for NRF2, pro-IL-1 $\beta$, mature IL-1 $\beta$, NLRP3, pro-caspase-1, caspase-1 p10, and gasdermin D. Equal amounts of protein from lysates or equal volumes of in-well lysates were loaded into the gel. Samples were run on SDS-polyacrylamide gels and transferred at $25 \mathrm{~V}$ onto nitrocellulose or PVDF membranes using a Trans-Blot ${ }^{\circledR}$ Turbo TransferTM System (Bio-Rad). The membranes were blocked in either $5 \% \mathrm{w} / \mathrm{v}$ milk or 2.5\% BSA (Sigma, A3608) in PBS, 0.1\% Tween 20 (PBST) for $1 \mathrm{~h}$ at room temperature. The membranes were then washed with PBST and incubated at $4^{\circ} \mathrm{C}$ overnight with goat anti-mouse IL-1 $\beta\left(250 \mathrm{ng} \mathrm{ml^{-1 }} ; \mathrm{R} \& \mathrm{D}\right.$ Systems, AF-401-NA), mouse anti-mouse NLRP3 (1 $\mu \mathrm{g} \mathrm{ml}^{-1}$; Adipogen, G-20B-0014-C100), rabbit anti-mouse caspase-1 (1.87 $\mu \mathrm{g} \mathrm{ml}^{-1}$; Abcam, ab179515), rabbit anti-mouse gasdermin D (0.6 $\mu \mathrm{g} \mathrm{ml}^{-1}$; Abcam, ab209845) or rabbit anti-mouse NRF2 (1.5 $\mu \mathrm{g} \mathrm{ml}^{-1}$; CST, 12721) primary antibodies in $0.1 \%$ (IL-1 $\beta$ ), 1\% (NLRP3) or 2.5\% (caspase-1, gasdermin D, NRF2) BSA in PBST. The membranes were washed and incubated with rabbit anti-goat IgG (500 $\mathrm{ng} \mathrm{ml}^{-1}, 5 \%$ milk in PBST; Dako, P044901-2), rabbit anti-mouse IgG $\left(1.3 \mu \mathrm{g} \mathrm{ml}^{-1}, 5 \%\right.$ milk in PBST; Dako, P026002-2) or goat anti-rabbit IgG (250 ng ml ${ }^{-1}, 2.5 \%$ BSA in PBST; Dako, (Dako, P044801-2) at room temperature for $1 \mathrm{~h}$. Proteins were then visualised with Amersham ECL Western Blotting Detection Reagent (GE Healthcare, RPN2236) and G:BOX (Syngene) and Genesys software. $\beta$-Actin (Sigma, A3854) was used as a

513 loading control. Densitometry was performed using FIJI (ImageJ). Uncropped western blots are 514 provided in Supplementary Figures 7-11.

ELISA

516 The levels of IL-1 $\beta$, IL-6 and tumour necrosis factor (TNF) in the supernatant were analysed by 517 enzyme-linked immunosorbent assay (ELISA; DuoSet, R\&D systems) according to the manufacturer's 518 instructions.

519 Cell death assays

520 Cell death was assessed by measuring lactate dehydrogenase (LDH) release into the supernatant using 521 a CytoTox 96 Non-Radioactive Cytotoxicity Assay (Promega) according to the manufacturer's 522 instructions. Cell death in OHSCs was assessed by adding propidium iodide (25 $\mu \mathrm{g} \mathrm{ml}^{-1}$; Sigma, $523 \mathrm{P} 4864)$ to the culture medium for the final $30 \mathrm{~min}$ of the inflammasome activation protocol followed 524 by widefield microscopy. 
526 ASC-citrine-expressing primary BMDMs were used to perform live imaging of ASC speck formation.

527 For itaconate derivative pre-treatment assays, cells were seeded at $1 \times 10^{6}$ cells ml $^{-1}$ in 96 -well plates

528 and incubated for $1 \mathrm{~h}$, and were then treated with vehicle (DMSO), DMI, 4OI or DMF (125 $\mu \mathrm{M}, 20 \mathrm{~h})$.

529 LPS $\left(1 \mu \mathrm{g} \mathrm{ml}^{-1}, 4 \mathrm{~h}\right)$ was then added to the wells to induce priming. The medium was replaced with

530 optimem, and nigericin $(10 \mu \mathrm{M})$ was added to activate the NLRP3 inflammasome. For assays where

531 itaconate derivative treatments were added after LPS priming, cells were seeded overnight at $1 \times 10^{6}$

532 cells ml $\mathrm{m}^{-1}$ in 96-well plates. Cells were then first primed with LPS $\left(1 \mu \mathrm{g} \mathrm{ml}^{-1}, 4 \mathrm{~h}\right)$. The medium was

533 replaced with optimum containing vehicle, DMI, 4OI or DMF (125 $\mu \mathrm{M}, 15 \mathrm{~min}$ ) prior to addition of

534 nigericin $(10 \mu \mathrm{M})$. Image acquisition began immediately after nigericin treatment. Images were

535 subsequently acquired every 10 min for a further 90 min using an IncuCyte ZOOM ${ }^{\circledR}$ Live Cell Analysis

536 system (Essen Bioscience) at $37^{\circ} \mathrm{C}$ using a 20X/0.61 S Plan Fluor objective. Speck number was

537 quantified using IncuCyte $Z \mathrm{OOM}^{\circledR}$ software, and was assessed for each treatment at the final time

538 point of $90 \mathrm{~min}$.

\section{OHSC immunostaining}

540 OHSCs were washed once with cold PBS and fixed in 4\% paraformaldehyde $(1 \mathrm{~h})$ at $4^{\circ} \mathrm{C}$. OHSCs 541 were washed two more times in cold PBS and then incubated with rabbit anti-mouse ASC $\left(202 \mathrm{ng} \mathrm{ml}^{-}\right.$

$542{ }^{1}$; CST, 67824) primary antibody overnight at $4^{\circ} \mathrm{C}$. OHSCs were washed and incubated with Alexa 543 Fluor $^{\mathrm{TM}} 488$ donkey anti-rabbit $\operatorname{IgG}\left(2 \mu \mathrm{g} \mathrm{m}^{-1}\right.$; Invitrogen, A-21206) secondary antibody for $2 \mathrm{~h}$ at 544 room temperature. All antibody incubations were performed using PBS, 0.3\% Triton X-100. Wash

545 steps were performed using PBST unless stated otherwise. OHSCs were washed and then incubated in 546 DAPI (1 $\mu \mathrm{g} \mathrm{ml}^{-1}, 15 \mathrm{~min}$; Sigma, D9542) at room temperature before final washing and mounting 547 using ProLong ${ }^{\mathrm{TM}}$ gold antifade mountant (Thermo, P36934) prior to imaging using widefield 548 microscopy.

Snapshot widefield microscopy

550 Images were collected on a Zeiss Axioimager.M2 upright microscope using a 5X or 20X Plan 551 Apochromat objective and captured using a Coolsnap HQ2 camera (Photometrics) through 552 Micromanager software (v1.4.23). Specific band-pass filter sets for DAPI and FITC were using to 553 prevent bleed-through from one channel to the next. 
555 Analysis was performed using FIJI (ImageJ) on images acquired from the same region of up to three 556 separate OHSCs (from the same insert) per treatment, and these values were averaged for each

557 biological repeat. ASC speck formation was quantified on $20 \mathrm{X}$ widefield microscopy images by 558 subtracting background (50 pixel rolling ball radius), manually setting thresholds and analysing 559 particles with the following parameters: size $1-10 \mu \mathrm{m}^{2}$, circularity $0.9-1.0$. To quantify PI uptake, 560 images were acquired on a widefield microscope using a $5 \mathrm{X}$ objective, background was subtracted (5.0 561 pixel rolling ball radius) and thresholds for images were automatically determined using the default 562 method. The total area of PI-positive signal was measured in the whole field of view, and was then 563 normalised to the total area of DAPI signal.

\section{Data analysis}

565 Data are presented as the mean \pm standard error of the mean (SEM) together with individual data points 566 where possible. Data were analysed using repeated-measures one-way or two-way analysis of variance 567 (ANOVA), or mixed effects model, with Dunnett's or Sidak's post-hoc test using GraphPad Prism 568 (v8). Transformations or corrections were applied as necessary to obtain equal variance between groups prior to analysis. Statistical significance was accepted at $* \mathrm{P}<0.05$.

\section{Acknowledgments}

571 ASC-citrine mice were a kind gift from Douglas Golenbock (University of Massachusetts Medical 572 School) and Te-Chen Tzeng (Bristol Myers-Squibb, Cambridge, USA). We thank Dr Kevin Stacey 573 (University of Manchester) for the routine isolation of human PBMCs.

\section{Competing interests}

575 The authors declare that the research was conducted in the absence of any commercial or financial 576 relationships that could be construed as a potential conflict of interest.

\section{$577 \quad 7 \quad$ Funding}

578 This work was funded by the MRC (Grant No. MR/N003586/1 to DB and SMA, and MR/T0116515/1 579 to DB) and was also funded by an MRC PhD studentship to CH (Grant No. MR/N013751/1). The 580 Bioimaging Facility microscopes used in this study were purchased with grants from BBSRC, 581 Wellcome Trust and the University of Manchester Strategic Fund. 


\section{Data availability}

583 The data that support the findings of this study are available from the corresponding author upon reasonable request.

\section{$9 \quad$ Author Contributions}

586 Conceptualisation: CH, EL, SMA and DB. Methodology: CH, EL and DB. Investigation: CH, EL and JPG. Writing - original draft preparation: $\mathrm{CH}$. Writing - review and editing: CH, EL, SMA and DB. Visualisation: CH. Supervision: EL, SMA and DB. Funding acquisition: DB and SMA.

10 References

Bambouskova, M., Gorvel, L., Lampropoulou, V., Sergushichev, A., Loginicheva, E., Johnson, K., Korenfeld, D., Mathyer, M. E., Kim, H., Huang, L. H., et al. (2018). Electrophilic properties of itaconate and derivatives regulate the I $\mathrm{KB} \zeta$-ATF3 inflammatory axis. Nature 556, 501-504.

Boucher, D., Monteleone, M., Coll, R. C., Chen, K. W., Ross, C. M., Teo, J. L., Gomez, G. A., Holley, C. L., Bierschenk, D., Stacey, K. J., et al. (2018). Caspase-1 self-cleavage is an intrinsic mechanism to terminate inflammasome activity. J. Exp. Med. 215, 827-840.

Chausse, B., Lewen, A., Poschet, G. and Kann, O. (2020). Selective inhibition of mitochondrial respiratory complexes controls the transition of microglia into a neurotoxic phenotype in situ. Brain. Behav. Immun. S0889-1591, 30209-9.

Chen, J. and Chen, Z. J. (2018). PtdIns4P on dispersed trans-Golgi network mediates NLRP3 inflammasome activation. Nature 564, 71-76.

Chen, G. Y. and Nuñez, G. (2010). Sterile inflammation: Sensing and reacting to damage. Nat. Rev. Immunol. 10, 826-837.

Coll, R. C., Robertson, A. A., Chae, J. J., Higgins, S. C., Muñoz-Planillo, R., Inserra, M. C., Vetter, I., Dungan, L. S., Monks, B. G., Stutz, A., et al. (2015). A small molecule inhibitor of the NLRP3 inflammasome for the treatment of inflammatory diseases. Nat Med 21, 248-255.

Cunha, M. I., Su, M., Cantuti-Castelvetri, L., Müller, S. A., Schifferer, M., Djannatian, M., Alexopoulos, I., van der Meer, F., Winkler, A., van Ham, T. J., et al. (2020). Proinflammatory activation following demyelination is required for myelin clearance and oligodendrogenesis. J. Exp. Med. 217, e20191390.

614 ElAzzouny, M., Tom, C. T. M. B., Evans, C. R., Olson, L. L., Tanga, M. J., Gallagher, K. A., 
615

616

617

618

619

620

621

622

623

624

625

626

627

628

629

630

631

632

633

634

635

636

637

638

639

640

641

642

643

Martin, B. R. and Burant, C. F. (2017). Dimethyl itaconate is not metabolized into itaconate intracellularly. J. Biol. Chem. 292, 4766-4769.

Fox, R. J., Miller, D. H., Phillips, J. T., Hutchinson, M., Havrdova, E., Kita, M., Yang, M., Raghupathi, K., Novas, M., Sweetser, M. T., et al. (2012). Placebo-Controlled Phase 3 Study of Oral BG-12 or Glatiramer in Multiple Sclerosis. N. Engl. J. Med. 367, 1087-1097.

Freeman, L., Guo, H., David, C. N., Brickey, W. J., Jha, S. and Ting, J. P. Y. (2017). NLR members NLRC4 and NLRP3 mediate sterile inflammasome activation in microglia and astrocytes. J. Exp. Med. 214, 1351-1370.

Gaidt, M. M., Ebert, T. S., Chauhan, D., Schmidt, T., Schmid-Burgk, J. L., Rapino, F., Robertson, A. A. B., Cooper, M. A., Graf, T. and Hornung, V. (2016). Human Monocytes Engage an Alternative Inflammasome Pathway. Immunity 44, 833-846.

Garstkiewicz, M., Strittmatter, G. E., Grossi, S., Sand, J., Fenini, G., Werner, S., French, L. E. and Beer, H. D. (2017). Opposing effects of Nrf2 and Nrf2-activating compounds on the NLRP3 inflammasome independent of Nrf2-mediated gene expression. Eur. J. Immunol. 47, 806-817.

Gold, R., Linker, R. A. and Stangel, M. (2012a). Fumaric acid and its esters: An emerging treatment for multiple sclerosis with antioxidative mechanism of action. Clin. Immunol. 142, 44-48.

Gold, R., Kappos, L., Arnold, D. L., Bar-Or, A., Giovannoni, G., Selmaj, K., Tornatore, C., Sweetser, M. T., Yang, M., Sheikh, S. I., et al. (2012b). Placebo-Controlled Phase 3 Study of Oral BG-12 for Relapsing Multiple Sclerosis. N. Engl. J. Med. 367, 1098-1107.

Gris, D., Ye, Z., Iocca, H. A., Wen, H., Craven, R. R., Gris, P., Huang, M., Schneider, M., Miller, S. D. and Ting, J. P.-Y. (2010). NLRP3 plays a critical role in the development of experimental autoimmune encephalomyelitis by mediating Th1 and Th17 responses. $J$. Immunol. 185, 974-81.

Groß, C. J., Mishra, R., Schneider, K. S., Médard, G., Wettmarshausen, J., Dittlein, D. C., Shi, H., Gorka, O., Koenig, P.-A., Fromm, S., et al. (2016). K+ Efflux-Independent NLRP3 Inflammasome Activation by Small Molecules Targeting Mitochondria. Immunity 45, 761-773.

Hall, S. M. (1972). The Effect of Injections of Lysophosphatidyl Choline into White Matter of the Adult Mouse Spinal Cord. J. Cell Sci. 10,.

Halle, A., Hornung, V., Petzold, G. C., Stewart, C. R., Monks, B. G., Reinheckel, T., Fitzgerald, K. A., Latz, E., Moore, K. J. and Golenbock, D. T. (2008). The NALP3 inflammasome is involved in the innate immune response to amyloid-beta. Nat Immunol 9, 857-865.

He, W.-T., Wan, H., Hu, L., Chen, P., Wang, X., Huang, Z., Yang, Z.-H., Zhong, C.-Q. and Han, J. (2015). Gasdermin D is an executor of pyroptosis and required for interleukin-1 $\beta$ secretion. Cell Res. 25, 1285-98.

Hooftman, A., Angiari, S., Hester, S., Corcoran, S. E., Runtsch, M. C., Ling, C., Ruzek, M. C., Slivka, P. F., McGettrick, A. F., Banahan, K., et al. (2020). The Immunomodulatory 
Metabolite Itaconate Modifies NLRP3 and Inhibits Inflammasome Activation. Cell Metab. 32, 468-478.e7.

Hornung, V., Bauernfeind, F., Halle, A., Samstad, E. O., Kono, H., Rock, K. L., Fitzgerald, K. A. and Latz, E. (2008). Silica crystals and aluminum salts activate the NALP3 inflammasome through phagosomal destabilization. Nat. Immunol. 9, 847-856.

Hoyle, C., Redondo-Castro, E., Cook, J., Tzeng, T., Allan, S. M., Brough, D. and Lemarchand, E. (2020). Hallmarks of NLRP3 inflammasome activation are observed in organotypic hippocampal slice culture. Immunology imm.13221.

Humphries, F., Shmuel-Galia, L., Ketelut-Carneiro, N., Li, S., Wang, B., Nemmara, V. V., Wilson, R., Jiang, Z., Khalighinejad, F., Muneeruddin, K., et al. (2020). Succination inactivates gasdermin D and blocks pyroptosis. Science (80-. ). 369, 1633-1637.

Jha, S., Srivastava, S. Y., Brickey, W. J., Iocca, H., Toews, A., Morrison, J. P., Chen, V. S., Gris, D., Matsushima, G. K. and Ting, J. P. Y. (2010). The inflammasome sensor, NLRP3, regulates CNS inflammation and demyelination via caspase-1 and interleukin-18. J. Neurosci. 30, 15811-15820.

Kayagaki, N., Warming, S., Lamkanfi, M., Walle, L. Vande, Louie, S., Dong, J., Newton, K., Qu, Y., Liu, J., Heldens, S., et al. (2011). Non-canonical inflammasome activation targets caspase-11. Nature 479, 117-121.

Kobayashi, E. H., Suzuki, T., Funayama, R., Nagashima, T., Hayashi, M., Sekine, H., Tanaka, N., Moriguchi, T., Motohashi, H., Nakayama, K., et al. (2016). Nrf2 suppresses macrophage inflammatory response by blocking proinflammatory cytokine transcription. Nat. Commun. 7, 11624.

Kornberg, M. D., Bhargava, P., Kim, P. M., Putluri, V., Snowman, A. M., Putluri, N., Calabresi, P. A. and Snyder, S. H. (2018). Dimethyl fumarate targets GAPDH and aerobic glycolysis to modulate immunity. Science (80-. ). 360, 449-453.

Kuo, P. C., Weng, W. T., Scofield, B. A., Paraiso, H. C., Brown, D. A., Wang, P. Y., Yu, I. C. and Yen, J. H. (2020a). Dimethyl itaconate, an itaconate derivative, exhibits immunomodulatory effects on neuroinflammation in experimental autoimmune encephalomyelitis. J. Neuroinflammation 17,.

Kuo, P.-C., Weng, W.-T., Scofield, B. A., Furnas, D., Paraiso, H. C., Yu, I.-C. and Yen, J.-H. (2020b). Induction of IRG1 following ischemic stroke promotes HO-1 and BDNF expression to alleviate neuroinflammation and restrain ischemic brain injury. J. Neuroinflammation.

Lassmann, H. and Bradl, M. (2017). Multiple sclerosis: experimental models and reality. Acta Neuropathol. 133, 223-244.

Law, S. H., Chan, M. L., Marathe, G. K., Parveen, F., Chen, C. H. and Ke, L. Y. (2019). An updated review of lysophosphatidylcholine metabolism in human diseases. Int. J. Mol. Sci. 20, 1149.

Lemarchand, E., Barrington, J., Chenery, A., Haley, M., Coutts, G., Allen, J. E., Allan, S. M. 
Li, S., Wu, Y., Yang, D., Wu, C., Ma, C., Liu, X., Moynagh, P. N., Wang, B., Hu, G. and Yang, S. (2019). Gasdermin D in peripheral myeloid cells drives neuroinflammation in experimental autoimmune encephalomyelitis. J. Exp. Med. 216, 2562-2581.

Liao, S. T., Han, C., Xu, D. Q., Fu, X. W., Wang, J. S. and Kong, L. Y. (2019). 4-Octyl itaconate inhibits aerobic glycolysis by targeting GAPDH to exert anti-inflammatory effects. Nat. Commun. 10, 5091.

Linker, R. A., Lee, D. H., Ryan, S., Van Dam, A. M., Conrad, R., Bista, P., Zeng, W., Hronowsky, X., Buko, A., Chollate, S., et al. (2011). Fumaric acid esters exert neuroprotective effects in neuroinflammation via activation of the Nrf2 antioxidant pathway. Brain 134, 678692.

Litjens, N. H. R., Burggraaf, J., Van Strijen, E., Van Gulpen, C., Mattie, H., Schoemaker, R. C., Van Dissel, J. T., Thio, H. B. and Nibbering, P. H. (2004). Pharmacokinetics of oral fumarates in healthy subjects. Br. J. Clin. Pharmacol. 58, 429-432.

Mills, E. and O'Neill, L. A. J. (2014). Succinate: A metabolic signal in inflammation. Trends Cell Biol. 24, 313-320.

Mills, E. L., Ryan, D. G., Prag, H. a., Dikovskaya, D., Menon, D., Zaslona, Z., Jedrychowski, M. P., Costa, A. S. H., Higgins, M., Hams, E., et al. (2018). Itaconate is an anti-inflammatory metabolite that activates Nrf2 via alkylation of KEAP1. Nature 556, 113-117. (2013). K+ Efflux Is the Common Trigger of NLRP3 Inflammasome Activation by Bacterial Toxins and Particulate Matter. Immunity 38, 1142-1153.

Nair, S., Huynh, J. P., Lampropoulou, V., Loginicheva, E., Esaulova, E., Gounder, A. P., Boon, 
A. C. M., Schwarzkopf, E. A., Bradstreet, T. R., Edelson, B. T., et al. (2018). Irg1 expression in myeloid cells prevents immunopathology during M. tuberculosis infection. J. Exp. Med. 215, 1035-1045.

O’Neill, L. A. J. and Artyomov, M. N. (2019). Itaconate: the poster child of metabolic reprogramming in macrophage function. Nat. Rev. Immunol. 19, 273-281.

Olagnier, D., Farahani, E., Thyrsted, J., Blay-Cadanet, J., Herengt, A., Idorn, M., Hait, A., Hernaez, B., Knudsen, A., Iversen, M. B., et al. (2020). SARS-CoV2-mediated suppression of NRF2-signaling reveals potent antiviral and anti-inflammatory activity of 4-octyl-itaconate and dimethyl fumarate. Nat. Commun. 11, 4938.

Perregaux, D. and Gabel, C. a. (1994). Interleukin-1 $\beta$ maturation and release in response to ATP and nigericin. Evidence that potassium depletion mediated by these agents is a necessary and common feature of their activity. J. Biol. Chem. 269, 15195-15203.

Pinteaux, E., Parker, L. C., Rothwell, N. J. and Luheshi, G. N. (2002). Expression of interleukin1 receptors and their role in interleukin-1 actions in murine microglial cells. J. Neurochem. 83, 754-763.

Plemel, J. R., Michaels, N. J., Weishaupt, N., Caprariello, A. V., Keough, M. B., Rogers, J. A., Yukseloglu, A., Lim, J., Patel, V. V., Rawji, K. S., et al. (2018). Mechanisms of lysophosphatidylcholine-induced demyelination: A primary lipid disrupting myelinopathy. Glia 66, 327-347.

Qin, W., Zhang, Y., Tang, H., Liu, D., Chen, Y., Liu, Y. and Wang, C. (2020). Chemoproteomic Profiling of Itaconation by Bioorthogonal Probes in Inflammatory Macrophages. J. Am. Chem. Soc. 142, 10894-10898.

Rock, K. L., Latz, E., Ontiveros, F. and Kono, H. (2010). The sterile inflammatory response. Annu. Rev. Immunol. 28, 321-42.

Schaum, N., Karkanias, J., Neff, N. F., May, A. P., Quake, S. R., Wyss-Coray, T., Darmanis, S., Batson, J., Botvinnik, O., Chen, M. B., et al. (2018). Single-cell transcriptomics of 20 mouse organs creates a Tabula Muris. Nature 562, 367-372.

Schroder, K. and Tschopp, J. (2010). The Inflammasomes. Cell 140, 821-832.

Schulze-Topphoff, U., Varrin-Doyer, M., Pekarek, K., Spencer, C. M., Shetty, A., Sagan, S. A., Cree, B. A. C., Sobel, R. A., Wipke, B. T., Steinman, L., et al. (2016). Dimethyl fumarate treatment induces adaptive and innate immune modulation independent of Nrf2. Proc. Natl. Acad. Sci. U. S. A. 113, 4777-4782.

Seoane, P. I., Lee, B., Hoyle, C., Yu, S., Lopez-Castejon, G., Lowe, M. and Brough, D. (2020). The NLRP3-inflammasome as a sensor of organelle dysfunction. J. Cell Biol. 219, e202006194.

Sheppard, O., Coleman, M. P. and Durrant, C. S. (2019). Lipopolysaccharide-induced neuroinflammation induces presynaptic disruption through a direct action on brain tissue involving microglia-derived interleukin 1 beta. J. Neuroinflammation 16, 1-13. 
Smith, D. (2017). Fumaric acid esters for psoriasis: a systematic review. Ir. J. Med. Sci. 186, 161-

Stoppini, L., Buchs, P. and Muller, D. (1991). A simple method for organotypic cultures of nervous tissue. J. Neurosci. Methods 37, 173-182.

Swain, A., Bambouskova, M., Kim, H., Andhey, P. S., Duncan, D., Auclair, K., Chubukov, V., Simons, D. M., Roddy, T. P., Stewart, K. M., et al. (2020). Comparative evaluation of itaconate and its derivatives reveals divergent inflammasome and type I interferon regulation in macrophages. Nat. Metab. 1-9.

Tannahill, G., Curtis, A., Adamik, J., Palsson-McDermott, E., McGettrick, A., Goel, G., Frezza, C., Bernard, N., Kelly, B., Foley, N., et al. (2013). Succinate is an inflammatory signal that induces IL-1 $\beta$ via HIF-1 $\alpha$. Nature 496, 238-242.

Trotter, A., Anstadt, E., Clark, R. B., Nichols, F., Dwivedi, A., Aung, K. and Cervantes, J. L. (2019). The role of phospholipase A2 in multiple Sclerosis: A systematic review and metaanalysis. Mult. Scler. Relat. Disord. 27, 206-213.

Tzeng, T. C., Schattgen, S., Monks, B., Wang, D., Cerny, A., Latz, E., Fitzgerald, K. and Golenbock, D. T. (2016). A Fluorescent Reporter Mouse for Inflammasome Assembly Demonstrates an Important Role for Cell-Bound and Free ASC Specks during In Vivo Infection. Cell Rep. 16, 571-582.

Wilms, H., Sievers, J., Rickert, U., Rostami-Yazdi, M., Mrowietz, U. and Lucius, R. (2010). Dimethylfumarate inhibits microglial and astrocytic inflammation by suppressing the synthesis of nitric oxide, IL-1 $\beta$, TNF- $\alpha$ and IL-6 in an in-vitro model of brain inflammation. $J$. Neuroinflammation 7, 30 .

Zhang, Y., Chen, K., Sloan, S. A., Bennett, M. L., Scholze, A. R., O'Keeffe, S., Phatnani, H. P., Guarnieri, P., Caneda, C., Ruderisch, N., et al. (2014). An RNA-Sequencing Transcriptome and Splicing Database of Glia, Neurons, and Vascular Cells of the Cerebral Cortex. J. Neurosci. 34, 11929-11947. 